Research Article

\title{
Theoretical and Experimental Comparison Results of Dual-Channel 3D Quasi-Optical Network System between Frequency Selective Surface and Wire Grid Polarizer
}

\author{
Hai Wang $\mathbb{D}^{1},{ }^{1}$ Xiaoming Liu, ${ }^{2}$ Junsheng Yu $\mathbb{D},{ }^{1}$ Zejian $L u,{ }^{3}$ and Xiaodong Chen ${ }^{4}$ \\ ${ }^{1}$ BUPT-QMUL EM Theory and Application International Research Lab, Beijing University of Posts and Telecommunications, \\ Beijing 100876, China \\ ${ }^{2}$ College of Physics and Electronic Information, Anhui Normal University, Wuhu, Anhui Province 241002, China \\ ${ }^{3}$ China Academy of Electronics and Information Technology, Beijing 100041, China \\ ${ }^{4}$ School of Electronic Engineering and Computer Science, Queen Mary University of London, London E1 4NS, UK
}

Correspondence should be addressed to Junsheng Yu; jsyu@bupt.edu.cn

Received 14 July 2017; Revised 20 November 2017; Accepted 12 December 2017; Published 26 March 2018

Academic Editor: Stefania Bonafoni

Copyright (C) 2018 Hai Wang et al. This is an open access article distributed under the Creative Commons Attribution License, which permits unrestricted use, distribution, and reproduction in any medium, provided the original work is properly cited.

A dual-channel 3D quasi-optical network (QON) system is analyzed and compared by theoretical and experimental results. It is divided into dual-channel signals by frequency selective surface (FSS) or wire grid polarizer (WGP) for transmitting the $324 \mathrm{GHz}$ signal on the top layer while diverting the $183 \mathrm{GHz}$ signal to the bottom layer. The system structure based on the beam radius is traced by the complex beam parameter and system transfer matrix for deciding the positions of cascade mirrors. The design principles and test results of FSS with perforated hexagonal array and WGP printed on the dielectric substrate are discussed together. In order to evaluate channel performance, the output planar near-fields of QON system are simulated and tested by the four reflections and three transmission results, respectively, where both scalar and vector conversion efficiencies are used to evaluate the quality of output beams. For the distortion phenomenon and energy loss in planar field, the aperture modes of corrugated horn feeds are analyzed by spherical wave expansion (SWE) to explain the asymmetrical pattern characteristic. The cut section of the far-field pattern is also directly measured in the compact antenna test range (CATR), comparing with the far-field patterns that are indirectly obtained based on the principle of near-to-far-field transformation and ideal aperture field integral of quiet zone (QZ). The differences between the near- and far-field test in QON have been compared, and discussion that the corresponding experimental results verify the WGP has better work performance in the good pattern symmetry, high cross-polarization isolation, low energy loss, and side-lobe level.

\section{Introduction}

In recent years, along with the rapid development of millimeter wave (MMW) and submillimeter wave technology, the QON system with the features of multifrequency and multipolarization is widely applied in the radio astronomy, meteorological satellites, remote sensing, and imaging system [1]. For example, the QO demultiplexer in MASTER has three FSS and one polarizer to perform the separation of five bands for measuring the atmospheric chemistry objectives [2]. The MWHS in FY-3A meteorological satellite provides vertical information about water vapor, which has five frequency and dual polarizations at 150 and $183.31 \mathrm{GHz}$ to monitor natural disasters $[3,4]$. A wire grid in the radioastronomy interferometer of ALMA Band 10 has been used to separate the two linear polarizations fields after two elliptical mirrors into corrugated horns, where the cross-polarization is lower than $-28 \mathrm{~dB}$ at all measured frequencies [5]. The channels of demultiplexing and filtering are also performed using FSS and polarizing grid in the MARSCHALS with three center bands at 300,325 , and $345 \mathrm{GHz}$ when the incident angle has $45^{\circ}[6]$ and so on. On the whole, their development 
mainly benefits from the progress of optic components and the application of Gaussian beam (GB) model analysis method $[7,8]$.

In the MMW and sub-MMW band, the atmosphere has the absorption characteristic when the electromagnetic wave propagates forward. The QON system often works at the peak absorption frequencies to measure those compositions on the microwave radiometer, such as the water vapor absorption lines have some center frequencies at 183.310, 323.826 , and $325.152 \mathrm{GHz}$ [9-12]. In order to integrate more channels in a very compact space, the design of QON system often selects double-layer structure that involves the separation, guidance, and bunching of beams with the help of a serious of feed horns, different cascaded mirrors, and filters, which makes it possible that all channel signals with different frequencies and polarizations can mechanically share a single main scanning reflector to satisfy satellite payload constraints on cost [13].

Typically, the feed in QON systems often selects the circular corrugated horn. It can finish a transformation of circular waveguide mode from fundamental $\mathrm{TE}_{11}$ mode to balanced hybrid $\mathrm{HE}_{11}$ mode in the horn aperture, where the aperture field has the characteristics of very low crosspolarization level and circular symmetrical radiation pattern with Gaussian distribution characteristic [14-17]. In the higher frequency band, limited by high machining accuracy in the grooves and junction, the machining quality of corrugated horn can easily affect its far-field pattern that leads to nonuniformity symmetrical Gaussian distribution due to the decreasing of main mode $\mathrm{HE}_{11}$. This problem often appears on the manufacturing during the process of spark erosion, classical milling, and electroforming [18]. It can bring greater distortion and deformation of beam after passing cascade mirrors.

On the other hand, the FSS comprises periodic arrays of metallic patches (reflection) or apertures (transmission) that are fabricated in a conductor surface directly or printed on a dielectric-slab indirectly, where the different periodic structures contain center connected, loop, solid interior, or combination types. Although they can finish a frequency selection perfectly with low-pass or high-pass features, obtaining a narrow band in the MMW range and very rapid cut-off characteristic outside the working frequency band is difficult [19, 20], so it is necessary to introduce the polarizer due to its frequency irrelevant attribute.

The polarizing grid is mainly used to separate double signals with horizontal and vertical polarizations [21]. The most optimal structure is the freestanding grid with round wire for higher transmissivity, such as copper or tungsten wire. They can be wounded across a supporting metal frame and then glued in place just like being sandwiched between two frame halves [22], but it should accord with the requirement that ensures the accuracy in the mounting and positioning attachments. However, in the MMW and sub-MMW range, it is limited by the processing and manufacturing condition because the wire spacing $g<\lambda / 2$ and round radius $a<\lambda / 10$ for a good polarization discrimination, which leads to spending more time in the wire-drawing, stretched tight, and vulnerability when it has periodic array distribution in a compact space. Another selectable component consists of many parallel metallic strips and plays the same vital role in polarization processing. Those parallel metallic strips can be directly manufactured by chemistry corrosion technology or printed on a dielectric substrate following a photolithography process [23-25] and become easier to ensure the precision of surface. Although the introduction of dielectric substrate makes the transmission coefficient reduced and become related to frequency in a certain extent, the phenomenon of multiple reflections in the substrate can be solved analogously by an antireflection coating with smaller dielectric constant, where the thickness of dielectric substrate can choose the multiple of quarter of a wavelength. What is more, the cross isolation between both directions should be further discussed, which is related to the ratio of wire period and width.

The FSS and the polarizer should be tested in the QO test bench, where the transmitted and received feeds are simultaneously placed in the double focus of elliptical mirrors as a test system if the device under test is also put in the public focus place $[26,27]$. During the FSS and grid measurement, the multipath stray signal and mutual coupling effect often occur from some reflections or transmission influencing the measurement results, which can be solved by a timedomain analysis technology [28]. Meanwhile, the measurement of QON system is essential for assessing the overall system working performance. However, there is rarely information to describe such system test process both in the nearfield and far-field measurement, and the difference between the double methods should also be more discussed. Based on the advanced MMW and sub-MMW instruments in the BUPT, the far-field radiation patterns of the corrugated horns can be directly implemented in the tri-reflectors CATR [29-31]. The near-field of dual-channel QON system at the output position has been obtained by a planar scanning in the two dimensions, where the sampling space can be chosen as $d x=d y<\lambda / 2$ according to the Nyquist sampling theory; meanwhile, their far-field pattern of dual channels is also directly measured in the tri-CATR compared with nearto-far-field transformation by Fourier transform (FT) [32]. Because inevitable stray signals existed in the surroundings, another method using aperture field integral with ideal plane wave is adopted to evaluate the far-field performance [33].

This paper mainly focuses on the design principle, measurement, and contrast method towards the dual-channel 3D QON system between FSS and polarizer. This dual-layer mirror structure can transmit $324 \mathrm{GHz}$ channel on the top layer and reflect the $183 \mathrm{GHz}$ to the bottom layer. The double channels are finally synthesized into a light path and then tested in the output position by near- and far-field. The mirror system based on the tracing of beam radius is firstly presented. The mode contents of feeds in the horn aperture are analyzed by the spherical wave expansion (SWE) method for verifying the asymmetrical pattern characteristic and energy loss during the near-field measurement. The principle of FSS and polarizer and their measurement results are also discussed in Section 2. The direct near-field planar measurement and three kinds of far-field results are compared in Sections 3 and 4, respectively. The corresponding experimental 
results verify the WGP has better work performance in the good pattern symmetry, high cross-polarization isolation, low energy loss, and side-lobe level. At last, we share relevant design and testing experience about the QON system.

\section{The Design Principle of QON System}

The QON system mainly controls the change of beam radius and propagation direction. The conical corrugated horn feed can radiate the circular symmetrical GB. When the GB propagates forward from the waist $\omega_{0}$ near the phase center of horn aperture, the beam radius $\omega$ changes with the distance of $z$ and has a relationship of $w=w_{0} \sqrt{1+\left(\lambda z / \pi w_{0}^{2}\right)^{2}}$.

For controlling the size of beam radius during the whole propagation path, the reflector mirror is mainly treated as a focusing unit to converge the beam radius thereby forming a new waist through it. When the lengths of ellipsoidal mirror from its double focal points to the center of surface are expressed as RI and RE, its equivalent focal length $f$ can be expressed as $1 / f=1 / \mathrm{RI}+1 / \mathrm{RE}$. According to the paraxial approximation and the geometrical optic limit, ellipsoidal mirror can be treated as a thin lens, where the beam radius changes with input distance $d_{\text {in }}$ in the free space, followed by the lens with $f$, and finally forms a new waist in the output distance $d_{\text {out }}$. After passing the lens through the distance $d$, the system transfer matrix using ABCD law can be expressed as $[7,8]$

$$
M_{\text {ellipsoid }}=\left[\begin{array}{ll}
A & B \\
C & D
\end{array}\right]=\left[\begin{array}{ll}
1 & d \\
0 & 1
\end{array}\right]\left[\begin{array}{cc}
1 & 0 \\
-\frac{1}{f} & 1
\end{array}\right]\left[\begin{array}{cc}
1 & d_{\text {in }} \\
0 & 1
\end{array}\right],
$$

where $0 \leq d \leq d_{\text {out }}$. Due to the beam radiating from the waist position, when the complex beam parameter for the incident direction has $q_{\text {in }}=j z_{c}=j \pi w_{0}^{2} / \lambda$, the parameter for output beam at any position $d$ has

$$
q_{\text {out }}=\frac{A q_{\text {in }}+B}{C q_{\text {in }}+D}=\frac{(1-d / f) j z_{c}+d_{\text {in }}+d\left(1-d_{\text {in }} / f\right)}{\left(1-d_{\text {in }} / f\right)-j z_{c} / f} .
$$

Then the radius of output beam can be obtained by the expression of $w_{\text {out }}=\operatorname{sqrt}\left(\lambda / \pi / \operatorname{Im}\left(-1 / q_{\text {out }}\right)\right)$. This $\mathrm{ABCD}$ matrix technique provides a good estimate for the characterization of beam size at different positions throughout the whole QON system.

2.1. The Dual-Channel 3D QON System. The dual-channel 3D QON system is modeled and simulated in the commercial GRASP software. It is shown in Figure 1, where the double corrugated horn feeds, three elliptical mirrors, and one filter using FSS or polarizer are installed. The double corrugated feed horns of T-183G and T-324G are similarly placed on the one focus point of elliptical mirrors M1 and M2, respectively, with distance D1 and D2. The lengths of long and short axis in mirrors have $218.6 \mathrm{~mm}$ and $180.9 \mathrm{~mm}$, and the half rim of mirrors equals to $\operatorname{Rim} 1=\operatorname{Rim} 2=\operatorname{Rim} 3=35 \mathrm{~mm}=$ $21.3 \lambda_{183 \mathrm{G}}=37.8 \lambda_{324 \mathrm{G}}$.

When beams spread to the filter using FSS or polarizer with distance $D 3=172.1 \mathrm{~mm}$ and $D 4=162.86 \mathrm{~mm}$, the beam propagation direction of T-183G and $\mathrm{T}-324 \mathrm{G}$ is reflected and transmitted dividedly, where the reflection angle has $\theta=30^{\circ}$ and then the double beams are synthesized into an emergent field by sharing the same reflection mirror M3 with a distance D5 $=92.12 \mathrm{~mm}$.

The changes of beam radius are calculated by (1)-(2) and shown in Figure 2. The waists of feeds are $\omega_{0,183 \mathrm{G}}=$ $1.585 \lambda_{183 \mathrm{G}}$ at $183 \mathrm{GHz}$ and $\omega_{0,324 \mathrm{G}}=1.485 \lambda_{324 \mathrm{G}}$ at $324 \mathrm{GHz}$, and then the double beams arrive at the mirrors M1 and M2 with $\omega_{\mathrm{M} 2,183 \mathrm{G}}=9.57 \lambda_{183 \mathrm{G}}$ and $\omega_{\mathrm{M} 1,324 \mathrm{G}}=17.8 \lambda_{324 \mathrm{G}}$. After the reflection of the first elliptical mirror M1 or M2, the radiuses of output beams achieve the minimum value with $\omega_{0,183 \mathrm{G}}=2.62 \lambda_{183 \mathrm{G}}$ and $\omega_{0,324 \mathrm{G}}=2.69 \lambda_{324 \mathrm{G}}$ before the filter. At the position of filter, the beam radiuses become $\omega_{f, 183 \mathrm{G}}=3.86 \lambda_{183 \mathrm{G}}=6.32 \mathrm{~mm}$ and $\omega_{f, 324 \mathrm{G}}=5.13 \lambda_{324 \mathrm{G}}=$ $4.75 \mathrm{~mm}$. Then they are commonly propagated to the M3, where the beam radius has $\omega_{\mathrm{M} 3,183 \mathrm{G}}=9.98 \lambda_{183 \mathrm{G}}$ and $\omega_{\mathrm{M} 3,324 \mathrm{G}}=16.35 \lambda_{324 \mathrm{G}}$.

However, the double beam waists are not placed in one of the focus points of the next mirror M3, which brings a phenomenon of beam distortion due to existing higher-order Gauss-Hermite modes $\mathrm{GB}(0,1)$ and $\mathrm{GB}(0,3)$ [7]. The distortion parameter $U$ can be defined by $U=w_{M} \tan \left(\theta_{i}\right) /(2 \sqrt{2} f)$, and the reflected power of beam is given by $K_{f}=1-U^{2}$. A criterion of $K_{f} \geq 0.99$ requires that the diameter $D$ of the mirrors should be four times the beam radius of $\omega_{M}$ on the mirror surface, and it also needs to satisfy with a relationship of $f / D \geq 0.5$ at the case of an incident angle $\theta_{i} \leq 30^{\circ}$. In this dual-channel 3D QON system, the double beams passing the M3 have the distortion with $U_{183 \mathrm{G}}=6.69 \%$ and $U_{324 \mathrm{G}}=6.19 \%$, where the corresponding reflection power is $K_{f, 183 \mathrm{G}}=99.5514 \%$ and $K_{f, 324 \mathrm{G}}=99.6162 \%$, respectively.

2.2. The Corrugated Feed Horn. The corrugated feed horn plays a very important role in the development of QON systems with high characteristics that its radiation pattern is Gaussian shaped and offers the potential with high gain, axial symmetry, low cross-polarization, and wide bandwidth. Generally, the corrugated horn is mainly divided into three sections that contain mode converter, corrugated profile, and phase stability section, respectively [14]. The input waveguide only needs to be excited by quite pure $\mathrm{TE}_{11}$ mode, which has dissymmetrical copolarization field in the E-plane and $\mathrm{H}$ plane. The mode converter is required to finish the $\mathrm{TE}_{11}$-to$\mathrm{HE}_{11}$ mode conversion over a specified number of slots with a choice of variable-depth slot, ring-loaded slot, or variablepitch-to-width slot $[15,34]$. The theoretical knowledge comes from the circular waveguide mode in the horn aperture that can be achieved into the balance condition with hybrid mode that has zero cross-polar field and is marked as $\mathrm{HE}_{11}$ similar to $85 \% \times \mathrm{TE}_{11}+15 \% \times \mathrm{TM}_{11}$ approximatively, when the deep of slots changes from $\lambda / 2$ to $\lambda / 4$ at the center frequency [35-37]. The types of internal corrugated profiles can select linear, sinusoid, tangential, exponential, hyperbolic, or polynomial and so on. Nevertheless, the phase of both fields is not coincidence matching thus increases a phase stability period to achieve the field in phase at the aperture, where the phase stability section has a single-slot depth $[14,16]$. 


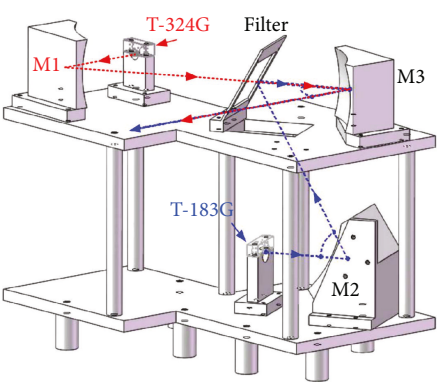

(a) The system view

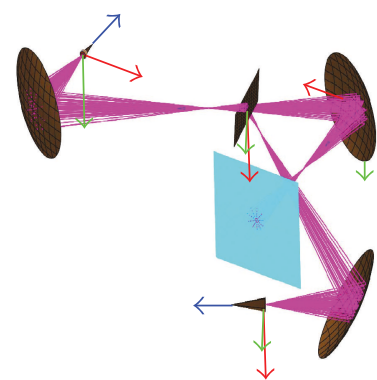

(b) The system light path

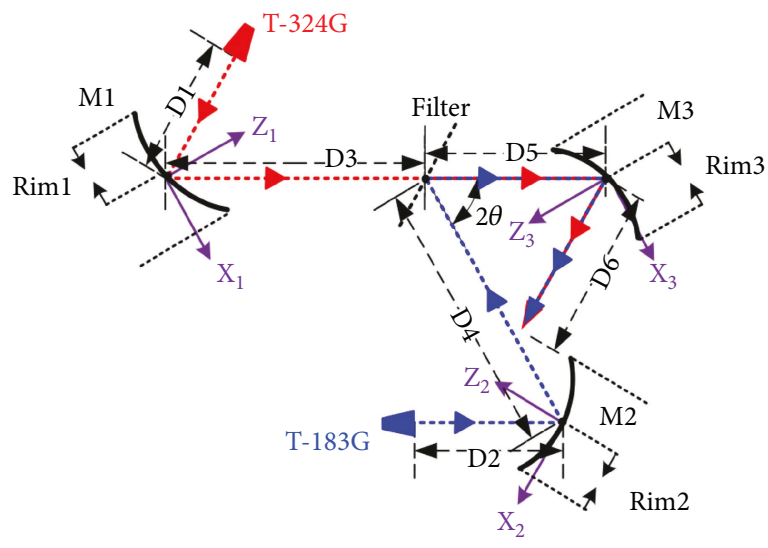

(c) The beam propagation distance

Figure 1: The dual-channel 3D QON system with $183 \mathrm{GHz}$ and $324 \mathrm{GHz}$.

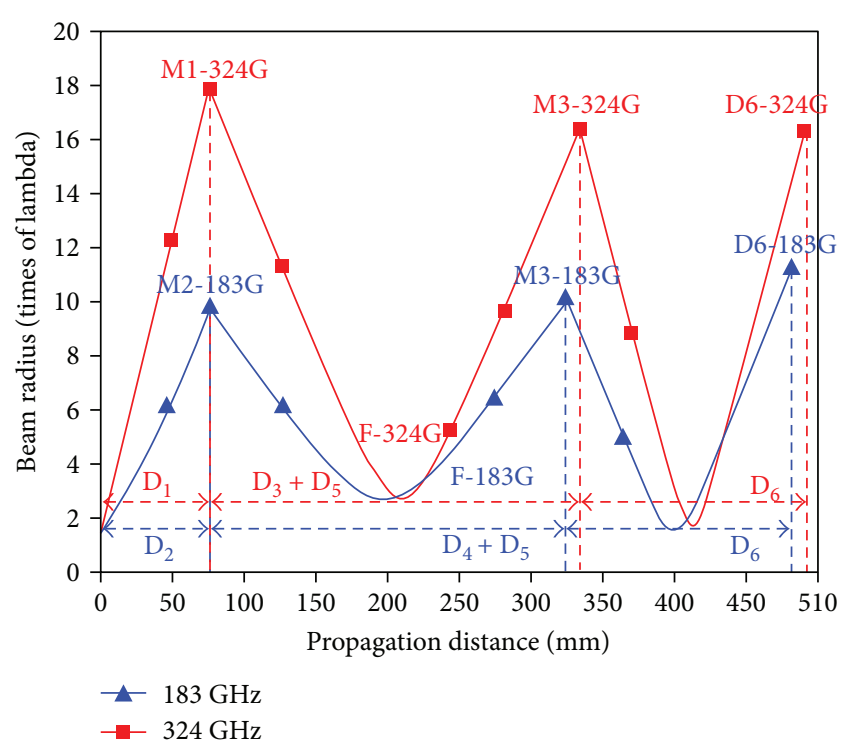

FIGURE 2: The changes of beam radius in the dual-channel 3D QON system.

In the 3D QON system, the double ultra-Gaussian corrugated feed horns with the cross of sine-squared and parallel section are designed and shown in Figures 3(a) and 3(b), respectively, where the profile section generates the $\mathrm{HE}_{11}$ and $\mathrm{HE}_{12}$ modes, and the parallel section brings them in phase [38]. The design principle comes from the circular waveguide mode matching technique, where the waveguide step discontinuity is analyzed by the scattering matrix and optimized in the CHAMP software for low side-lobe and cross-polar levels. The both WR4 and WR3 feeds after machining are directly measured by the tri-reflectors CATR in the BUPT $[30,39,40]$. The test results of co- and cxpolarization radiation field are presented in Figures 3(c) and $3(\mathrm{~d})$ working at the $183 \mathrm{GHz}$ and $324 \mathrm{GHz}$, where the side-lobe levels have $-39 \mathrm{~dB}$ and $-42.3 \mathrm{~dB}$, and the crosspolarizations are $-30 \mathrm{~dB}$ and $-29 \mathrm{~dB}$, respectively. Furthermore, by the spherical wave expansion (SWE) method, the mode components are obtained at the aperture of horns, where the spherical expansions expressed in the Q-modes with coefficients $Q_{\text {smn }}$ are written as $[41,42]$

$$
E(r, \theta, \phi)=k_{0} \sqrt{\eta_{0}} \sum_{s=1}^{2} \sum_{m=-M}^{M} \sum_{n=|m|>0}^{N(m)} Q_{s m n} F_{\text {smn }}(r, \theta, \phi),
$$

where the number of $\theta$ samples and $\phi$ samples over $360^{\circ}$ is $n_{\text {theta }}$ and $n_{\text {phi }}$. The spherical modes have an azimuthal index $m$ and a polar index $n$, the maximum polar index $N$ is selected as $1 \leq N_{\max } \leq n_{\text {theta }} / 2$, and the maximum azimuthal index $M$ is $0 \leq M_{\max } \leq \min \left\{\left(n_{\mathrm{phi}}-1\right) / 2, N_{\max }\right\}$. The $k_{0}$ and $\eta_{0}$ are the wavenumber $2 \pi / \lambda$ and free-space impedance. The $F_{\text {smn }}(r, \theta, \phi)$ represents the power-normalized spherical vector wave functions by the associated Legendre polynomials $\bar{P}_{n}^{|m|}(\cos \theta)$ and the spherical Hankel functions of the second kind $h_{n}^{2}(k r)$ [41]. 


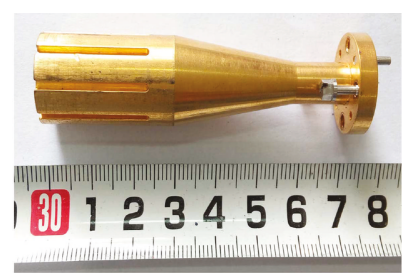

(a) WR4 feed

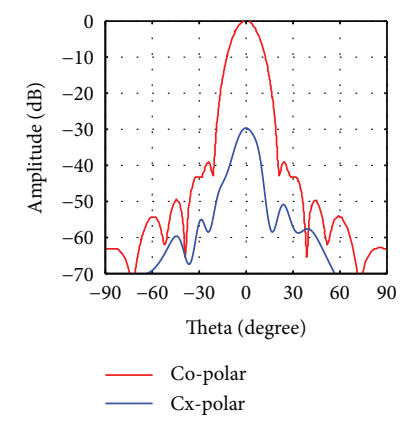

(c) Far-field of WR4 at $183 \mathrm{GHz}$
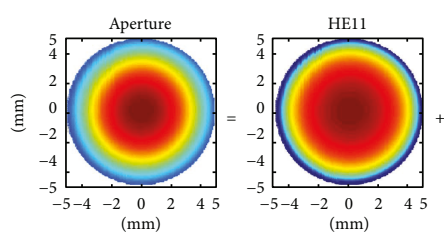

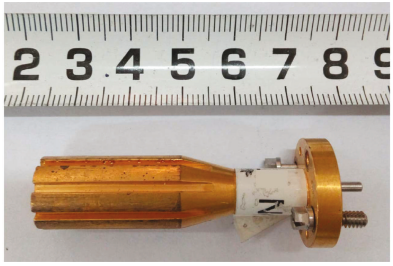

(b) WR3 feed

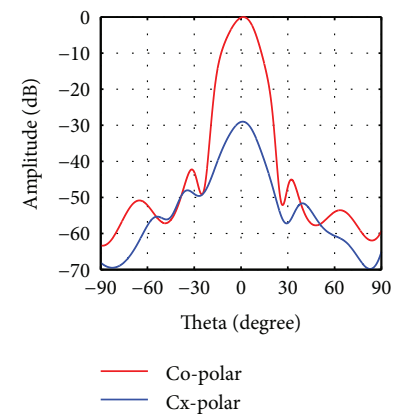

(d) Far-field of WR3 at $324 \mathrm{GHz}$
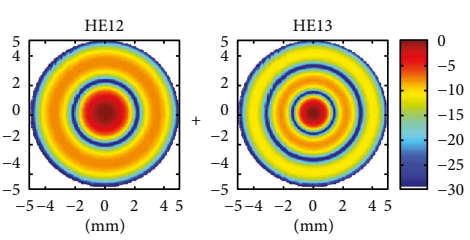

(e) Aperture field of WR4 feed at $183 \mathrm{GHz}$. From left to right are aperture field,
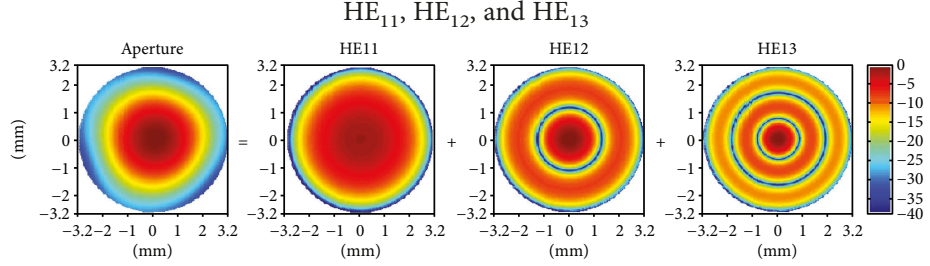

(f) Aperture field of WR3 feed at $324 \mathrm{GHz}$. From left to right are aperture field,

$$
\mathrm{HE}_{11}, \mathrm{HE}_{12} \text {, and } \mathrm{HE}_{13}
$$

FIgURE 3: The radiation far-field and aperture patterns of corrugated feed horns.

When the Q-mode coefficients $Q_{\mathrm{smn}}$ are solved, the tabulated SWE coefficients are fed as the tabulated feed. Then the aperture fields of double feed horns are obtained after passing own length of WR4 and WR3. The mode components of aperture field in the vector direction about WR4 at $183 \mathrm{GHz}$ and WR3 at $324 \mathrm{GHz}$ are calculated out and made up of $\mathrm{HE}_{11}: \mathrm{HE}_{12}: \mathrm{HE}_{13}=0.971: 0.032: 0.001$ and $\mathrm{HE}_{11}$ : $\mathrm{HE}_{12}: \mathrm{HE}_{13}=0.872: 0.128: 0.001$. The planar aperture field is also shown in Figures 3(e) and 3(f), where the WR4 horn has axis-symmetrical pattern features both in far-field and aperture field. Due to higher difficulty in the processing with the rise of frequency, the aperture field of the WR3 feed has some noncircular symmetric Gaussian decay characteristics in the amplitude, which is caused by the decreasing of the level of main mode $\mathrm{HE}_{11}$ and an increasing of another $\mathrm{HE}_{12}$ mode.

2.3. The FSS. The FSS in the dual-channel QON system selects a perforated metal plat plate with periodical circular hole array structure. This type of FSS has a bandpass filter characteristic, and the main design principle is the Floquet theory in the short circuit and the waveguide mode theory in the open circuit, where the minimum and the highest cut-off frequencies during the passband are determined by the cut-off frequency of waveguide unit and the higherorder Floquet modes, respectively [43]. For the case of normal incidence, the reflection and the transmission coefficients of the circular openings arranged in equilateral triangular lattice can be reduced to following forms

$$
T=\frac{1}{1-j[A+B \tanh (\beta h / 2)]}-\frac{1}{1-j[A+B \operatorname{coth}(\beta h / 2)]},
$$

$$
R=\frac{1}{1-j[A+B \tanh (\beta h / 2)]}+\frac{1}{1-j[A+B \operatorname{coth}(\beta h / 2)]},
$$


where $h$ is the thickness of metal plate, and the other parameters of $A, B$, and $\beta$ can be found in [43]. According to the transmission line theory, considering the circular hole as a circular waveguide, when the FSS needs to transmit $f_{c}=324 \mathrm{GHz}$, its cut-off lambda $\lambda_{c}$ is linked to the hole radius $a$ and satisfies the relationship with $a=\lambda_{c} / 3.41=0.27 \mathrm{~mm}$. From (4), if the spacing $d$ between adjacent holes becomes smaller, the transmission bandwidth of passband is wider. The thickness $h$ of FSS can influence the energy loss. What is more, it can further decrease the ripple and reflection coefficient in the passband, and the single thickness can select $h=\lambda_{c} / 5 \sim \lambda_{c} / 2$. The structure of FSS having circular hole and equilateral triangular lattice is shown in Figure 4(a), where it has the radius $a=0.26 \mathrm{~mm}$, the thickness $h=0.4 \mathrm{~mm}$, and the size $4 \mathrm{~cm} \times 4 \mathrm{~cm}$ and is adjacent to surrounding borders with $1 \mathrm{~cm}$ extension. Meanwhile, the incidence angle can influence its frequency response curve both in transmission and reflection but is not sensitive to incidence polarization wave, which are shown in Figures 4(b) and 4(c) at the angles of $0^{\circ}, 15^{\circ}, 30^{\circ}$, and $45^{\circ}$. The larger incidence angle is, the smaller transmission resonance frequency has. For the requirement of incidence angle $30^{\circ}$, the FSS needs to play a good role in the beam separation at the $183 / 324 \mathrm{GHz}$. The measured transmission coefficient at $30^{\circ}$ has $-30.21 \mathrm{~dB}$ at $183 \mathrm{GHz}$ and $-0.53 \mathrm{~dB}$ at $324 \mathrm{GHz}$ with vertical polarization incidence, comparing the simulation values with $-29.35 \mathrm{~dB}$ at $183 \mathrm{GHz}$ and $-0.16 \mathrm{~dB}$ at $324 \mathrm{GHz}$. However, due to the influence of coupling responses on two-feed radiation field and small structure size of holes, the reflection result is only given directly by simulation with $-0.011 \mathrm{~dB}$ at $183 \mathrm{GHz}$ and $-19.74 \mathrm{~dB}$ at $324 \mathrm{GHz}$ at $30^{\circ}$.

2.4. The Polarizer. In this QON system, the broadband WGP printed on the dielectric substrate is designed. The polarizing grid can reflect perfectly the field that electric vector parallels to the direction of grid wires and transmit without loss orthogonally polarized radiation field. The analysis method of grids with dielectric substrate can adopt the transmission line equivalent circuit. The shunt reactance of an inductive grid sandwiched between air and substrate having index of refraction $n_{1}$ and $n_{2}$, respectively, is [7]

$$
X_{i}=-\frac{2}{n_{1}^{2}+n_{2}^{2}} w_{0}^{\prime}\left(\frac{w}{w_{0}^{\prime}}-\frac{w_{0}^{\prime}}{w}\right)^{-1} \ln \csc \left(\frac{\pi g}{a}\right),
$$

where it has $w_{0}^{\prime}=w_{0} \sqrt{2 /\left(n_{1}^{2}+n_{2}^{2}\right)}, g$ and $2 a$ are the period and width of the polarizing grid.

According to the equivalent transmission line model in Figure 5, the reflection and transmission coefficients can be expressed as $R=\left(Z-Z_{s}\right) /\left(Z+Z_{s}\right)$ and $T=2 Z /\left(Z+Z_{s}\right)$, so the polarization isolation between both directions is related to the ratio of $g / 2 a$, and the final relation is shown in Figure 6 at different frequencies. Due to the machining becoming more difficult for small radius $a$, when the grid and the incident angle are fixed with $a=0.04 \mathrm{~mm}$ and $\theta_{i}=30^{\circ}$, the polarization isolation decreases with the increase of frequency and has the variation with a period of $g / 2 a=1$. Considering the actual processing precision in the printed circuit board (PCB), the pitch-to-width ratio of $g / 2 a$ should be selected at the range of $[3.2,3.8]$, where the theoretical polarization isolation can be less than $-42 \mathrm{~dB}$.

Meanwhile, for increasing the transmission coefficient, the phase delay $\Phi$ of reflected field on the double interfaces of substrate should satisfy a relationship with $\Phi=2 \beta d$ and equal to odd times of $\pi$, so the thickness of dielectric substrate should be selected as a quarter-wave matching layer [44]

$$
d_{\varepsilon}=\frac{\left(K_{0}\right) \lambda}{4 \cdot \sqrt{\varepsilon-\sin ^{2} \theta_{i}}},
$$

where $K_{0}$ is an odd integral value, the $\theta_{i}$ is the incident angle, and the propagation constant in the substrate has $\beta=(2 \pi / \lambda)$ $\left(\sqrt{n_{2}}-\sin ^{2} \theta\right)^{0.5}$. In the case of vertical incidence, it has minimum substrate thickness with $d_{\varepsilon, \min }=\lambda /(4 \sqrt{\varepsilon})$. The dielectric material selects PTFE with low dielectric constant for good transmission. The structure model of inductive strip grid is shown in Figure 7(a), where the size has $6 \mathrm{~cm} \times 6 \mathrm{~cm}$. The simulation of TM wave reflection and TE wave transmission and their polarization isolation at the different incidence angles are also shown in Figures 7(b) and 7(c), where the incidence angle has larger influence in the transmission coefficients than that in the reflection. The simulation of TM wave reflection coefficients is directly given that there are $0.022 \mathrm{~dB}$ at $183 \mathrm{GHz}$ and $-0.076 \mathrm{~dB}$ at $324 \mathrm{GHz}$, and the TE wave transmission coefficients have $-0.008 \mathrm{~dB}$ at $183 \mathrm{GHz}$ and $-0.36 \mathrm{~dB}$ at $324 \mathrm{GHz}$ for the incidence of $30^{\circ}$. The polarization isolation of TE wave transmission has been measured from $110 \mathrm{GHz}$ to $325 \mathrm{GHz}$ at the vertical incidence, where its isolation is processed by the time domain analysis technology for reducing the influence of stray wave signals and the multipath coupling effect, which are plotted in right axis of the Figure $7(\mathrm{c})$ and have $-25.33 \mathrm{~dB}$ at $183 \mathrm{GHz}$ (simulation is $-28.41 \mathrm{~dB}$ ) and $-19 \mathrm{~dB}$ at $324 \mathrm{GHz}$ (simulation is $-18.87 \mathrm{~dB}$ ) at $30^{\circ}$.

\section{Near-Field Planar Testing Results of QON System}

The near-field planar measurement of the dual-channel 3D QON system is implemented in the anechoic chamber. The test platform is shown in Figure 8 and is driven by the scanners with two-axis stepper motors. The magnitude and phase information of near-field are continuous sampling at regular intervals over a well-defined output plane with a size of $80 \mathrm{~mm} \times 80 \mathrm{~mm}$. Due to the fact that the maximal sampling interval of $\Delta x$ and $\Delta y$ is $\lambda / 2$ from the Nyquist sampling rate, the planar testing intervals are selected as being less than $\lambda / 2$ having $d x=d y=0.8 \mathrm{~mm}$ at $183 \mathrm{GHz}$ and $d x=d y=0.46 \mathrm{~mm}$ in $324 \mathrm{GHz}$. The measurement distance of near-field has D6 $=160 \mathrm{~mm}$ in both $183 \mathrm{GHz}$ and $324 \mathrm{GHz}$. Meanwhile, the both copolarization and cross-polarization fields are measured at the same time. When the filter selects the polarizer or FSS, the polarization direction of transmitted feed at $183 \mathrm{GHz}$ channel is rotated as horizontal polarization for 


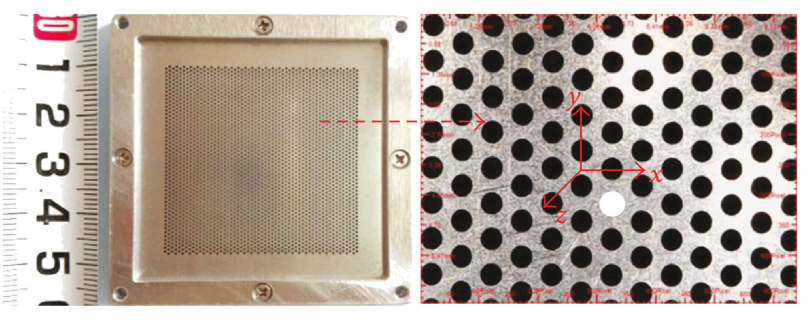

(a) Single-layer FSS has circular hole with equilateral triangular lattice

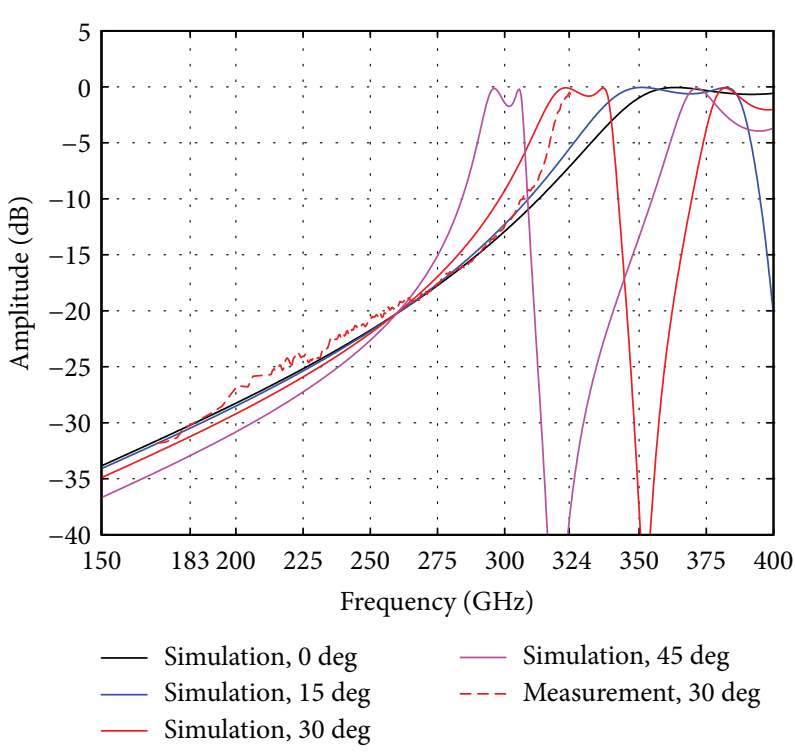

(b) The simulation and measurement results of transmission coefficients

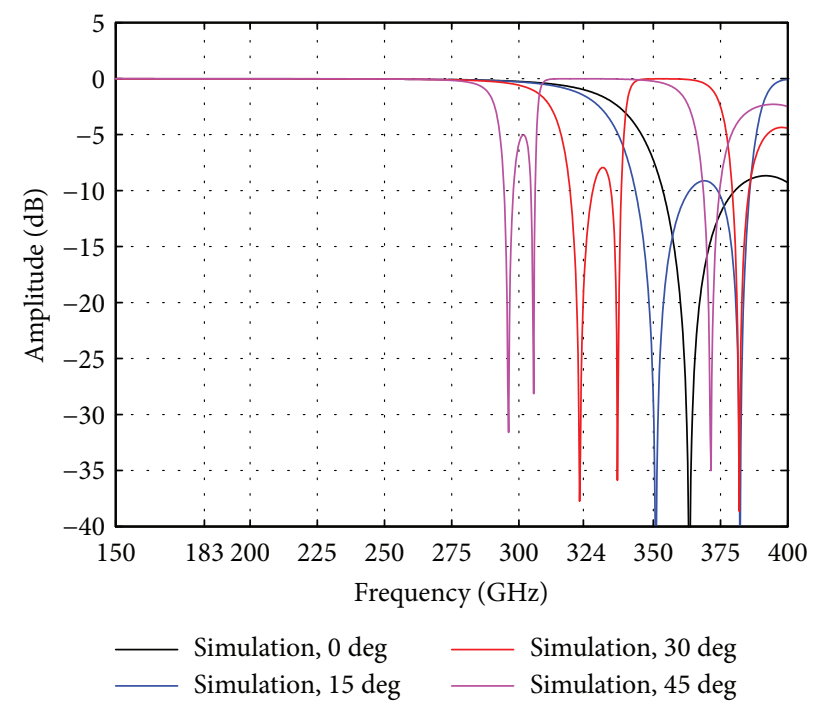

(c) The simulation results of reflection coefficients

FIGURE 4: The structure of FSS and its frequency response curve at the different incidence angles of $0^{\circ}, 15^{\circ}, 30^{\circ}$, and $45^{\circ}$.
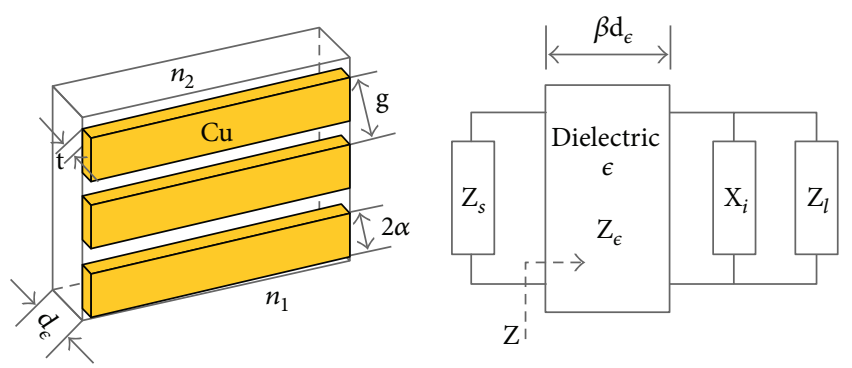

Figure 5: The structure of grid printed on the dielectric substrate and its equivalent transmission line model.

reflection, and otherwise it is vertical polarization for transmission at $324 \mathrm{GHz}$.

Of course, the simulation model of QON system is also created in the commercial GRASP software. The single unit cell of FSS with equilateral triangular lattice is the plane with a central circular hole and limited by a tabulated rim that is given by means of a tabulation data. The table values define many points along the rim, in a plane perpendicular to the $z$-axis of the coordinate system of FSS, and are tabulated in rectangular XOY coordinates. According to the size of beam radius calculated in the filter position, the dimension of FSS structure is selected as the $24.89 \mathrm{~mm} \times 24.89 \mathrm{~mm}$ and can satisfy the condition that the length is larger than the four-time beam radius both in double channels. There are a total of 529 units cell, and the single unit cell is defined by a rim in total of 2288 data points. Correspondingly, the polarizer is also established by the rectangular plate with electrical properties of strip grid and dielectric layer based on the simulation parameters. When the beam spreads to filter, the reflection fields of $183 \mathrm{GHz}$ are obtained by the rigorous current analysis using the physical optic (PO) method. 


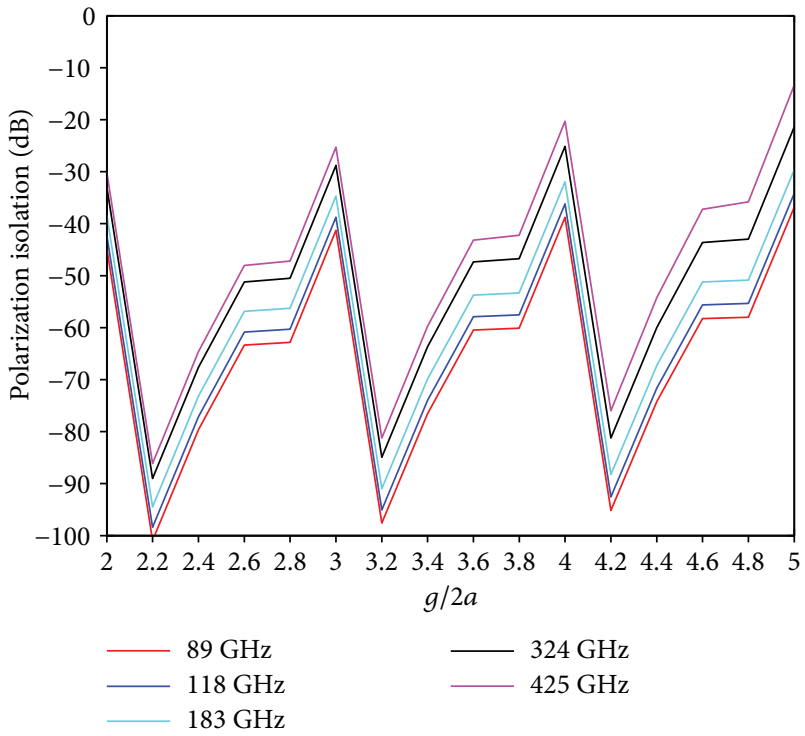

Figure 6: The relationship between pitch-to-width ratio $g / 2 a$ and polarization isolation $(\mathrm{dB})$ at different frequencies of $89 / 118 / 183$ / $324 / 425 \mathrm{GHz}$.

In order to compare the consistency among the different planar output fields, the scalar and vector conversion efficiency formulas $\eta_{s}$ and $\eta_{v}$ on behalf of beam energy distribution and vector direction are used. Especially, comparing with results of planar measurement $f_{1} \cdot \exp \left(j \varphi_{1}\right), 183 \mathrm{GHz}$ channel selects the measured result of aluminum plate and the $324 \mathrm{GHz}$ channel chooses the direct transmission through air as the reference field $f_{2} \cdot \exp \left(j \varphi_{2}\right)$.

$$
\begin{aligned}
& \eta_{s}= \frac{\iint_{S}\left|f_{1}\right| \cdot\left|f_{2}\right| \mathrm{ds}}{\sqrt{\iint_{S}\left|f_{1}\right|^{2} \mathrm{~d} s \cdot \iint_{S}\left|f_{2}\right|^{2} \mathrm{ds}}}, \\
& \eta_{v}=\frac{\iint_{S}\left|f_{1}\right|\left|f_{2}\right| \exp \left[j\left(\varphi_{1}-\varphi_{2}\right)\right] \mathrm{d} s \cdot \iint_{S}\left|f_{1}\right|\left|f_{2}\right| \exp \left[j\left(\varphi_{2}-\varphi_{1}\right)\right] \mathrm{d} s}{\iint_{S}\left|f_{1}\right|^{2} \mathrm{~d} s \cdot \iint_{S}\left|f_{2}\right|^{2} \mathrm{ds}} .
\end{aligned}
$$

3.1. The $183 \mathrm{GHz}$ Channel Near-Field Planar Measurement. The output planar near-field of $183 \mathrm{GHz}$ channel is measured in the four cases using different filters, including FSS, polarizer, and direct reflectors by copper or aluminum plate. Thereinto, the double direct reflectors are adapted to compare the energy loss by the system itself.

The testing planar grid has a total of $111 \times 111$ data number within the output range of $80 \mathrm{~cm} \times 80 \mathrm{~cm}$. The four testing results of two-dimension field are shown in Figure 9, where the amplitude and phase information of $\mathrm{co}^{-}$and cross-polarizer are given together. The more detailed parameters contain the cross-polarization level, the Gaussian beam radiuses, and the consistency of copolarization pattern between simulation and measurement and are summarized in Table 1.
In general, from the measurement results in Figures 9(a)9(d), the four copolarization fields have circular symmetry Gaussian distribution characteristic in both the amplitude and phase, which is similar to the radiation field of WR4 corrugated horn feed in Figure 3(e). The beam radius can be well controlled after passing through the double reflector mirrors and one filter. Inversely, the amplitude of cross-polarization has double peak levels during the whole planar beam ranges especially at the case of using polarizer. Their phases have the same Gaussian attenuation characteristic with the spiral shape, where the phase converges uniformly to the center position of beam. Furthermore, those radiation patterns of amplitude and phase have the same discontinuity-like ripples in the bottom. This phenomenon can be interpreted by the system diffraction from the structure framework when the received horn is moved to the edge of system baseplate.

For the cross-polarization level, comparing the simulation and measurement results given in Table 1 , the actual test levels are generally lower $3 \mathrm{~dB} \sim 4 \mathrm{~dB}$ than the simulation values. The test using FSS and polarizer can reach up to $-18.33 \mathrm{~dB}$ and $-18.82 \mathrm{~dB}$, respectively, which are close to the direct reflection by plane mirror using copper and aluminum plate with $-18.07 \mathrm{~dB}$ and $-17.76 \mathrm{~dB}$ separately.

The accurate calculation of beam radius takes the average position values of both sides at the E-field and $\mathrm{H}$-field. According to the simulation and measurement results, the four beam radiuses have a small difference relative to the theoretical radius value with $\omega_{\text {out }, 183 \mathrm{G}}=11.07 \lambda_{183 \mathrm{G}}$. This small change mainly comes from the uncertainty of $\mathrm{GB}$ waist position in the actual WR4 corrugated horn feed, the assembly location and angle errors of QON components, the shift of measuring planar position being far away from the center of M3 mirror, and the movement precision of scanning motors when it accurately reaches the test plane at the D6. Obviously, this difference of beam radius in D6 can reach the range from $2.89 \%$ to $5.03 \%$ relative to theoretical value seeing from all measurement at $183 \mathrm{GHz}$. For the most case of waist position, if the input distance D2 increases $+1 \mathrm{~mm}$ and $+2 \mathrm{~mm}$, the theoretical output radius in the D6 will increase $+0.4 \lambda_{183 \mathrm{G}}$ and $+0.8 \lambda_{183 \mathrm{G}}$, which are close to the simulation and measurement values.

In the beam consistency of copolarizer field, the scalar conversion efficiency has $\eta_{s \text {, polarizer }}=98.7954 \%$ relative to the reference result of aluminum plate with a $100 \%$. The FSS has also a similar result with $\eta_{s, \mathrm{FSS}}=98.9749 \%$. Although the double energy loss has exceeded $1 \%$ with respect to the direct reflection case just only choosing a different filter, the energy loss of reflection with $0.9 \%$ should be a common phenomenon in the testing of QON system based on another direct energy calculation using copper plate with $\eta_{s, \text { copper }}=99.0955 \%$.

Meanwhile, in the vector conversion efficiency, the values become lower for obtaining bigger influence from the difference of phase. The three different filters have the highest ratio up to a total of $\eta_{s \text {,polarizer }}=97.9952 \%$, but it has a contrast that the double ideal reflection between copper and aluminum plate is only $\eta_{s, \text { copper }}=97.5476 \%$. It further demonstrates that the filters using FSS with perforated array and polarizer with strip grids can play a perfect reflection performance in the 


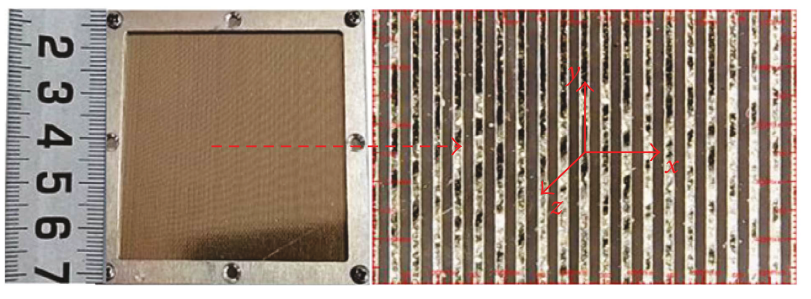

(a) The strip structure of WGP

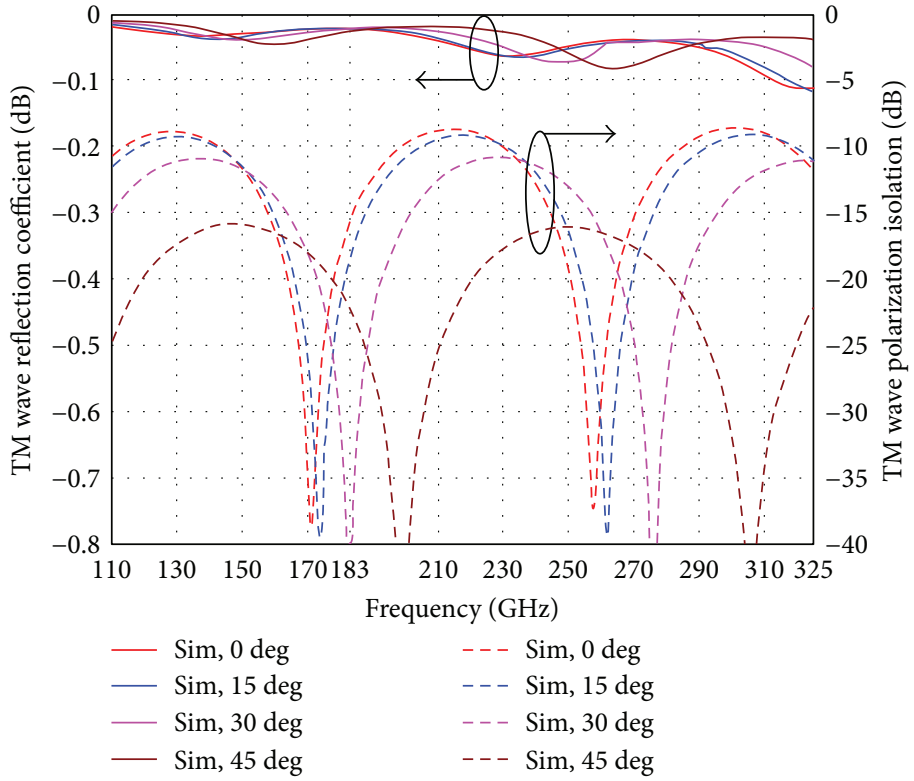

(b) The results of TM wave reflection and its polarization isolation
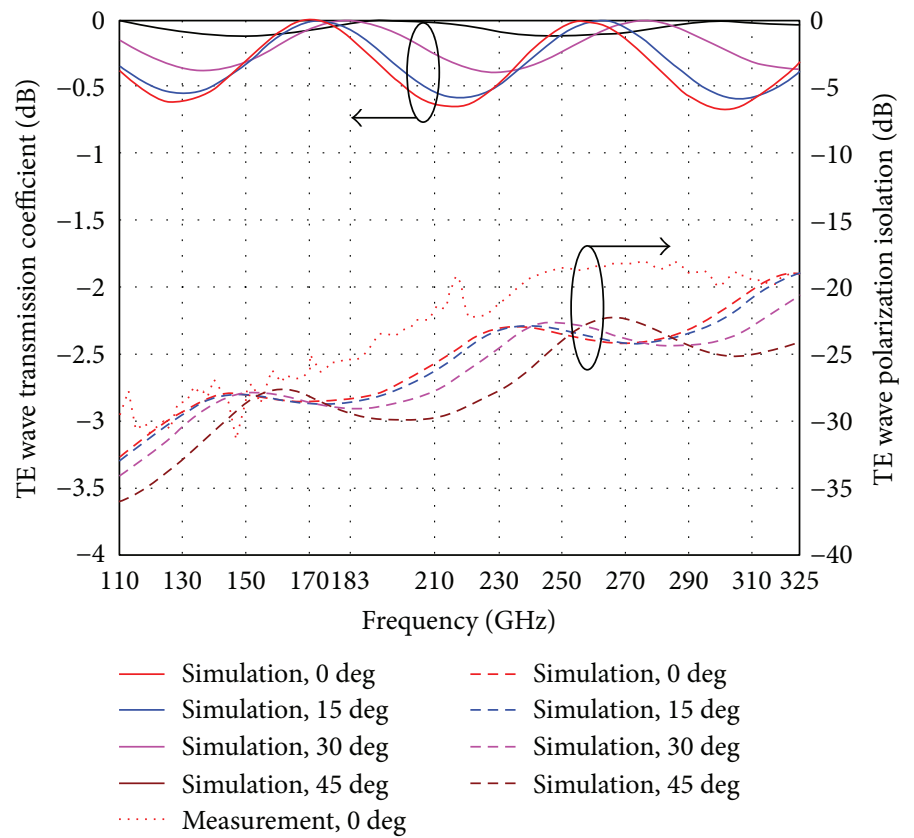

(c) The results of TE wave transmission and its polarization isolation

Figure 7: The structure model of WGP and its frequency response curve with different incidence angles of $0^{\circ}, 15^{\circ}, 30^{\circ}$, and $45^{\circ}$ at TE and TM wave.

$183 \mathrm{GHz}$ channel. Of course, at the same test conditions for beam consistency distribution, the influence from the variation of phase is bigger than that from the change of amplitude when different filters are adopted.

3.2. The $324 \mathrm{GHz}$ Channel Near-Field Planar Measurement. The output planar near-field of $324 \mathrm{GHz}$ channel on the front layer is similarly measured with the three different cases, including FSS, polarizer, and direct transmission through air as a reference. The planar grid has $201 \times 201$ data number in total within the output range of $80 \mathrm{~cm} \times 80 \mathrm{~cm}$, and those testing results are shown in Figure 10. The comparative values are also summarized in Table 2 . Due to the fact that the transmissive effect traversing the filter cannot be simulated in the software, the double output fields in simulation are calculated by the same PO currents when the beam passes through the FSS or polarizer at $324 \mathrm{GHz}$.

From the planar measurement results shown in Figures 10(b) and 10(c), the double fields have a small noncircular symmetric characteristic at the copolarization amplitude when the level of beam pattern is far away from the center of $-20 \mathrm{~dB}$, which is caused by the same nonuniform 


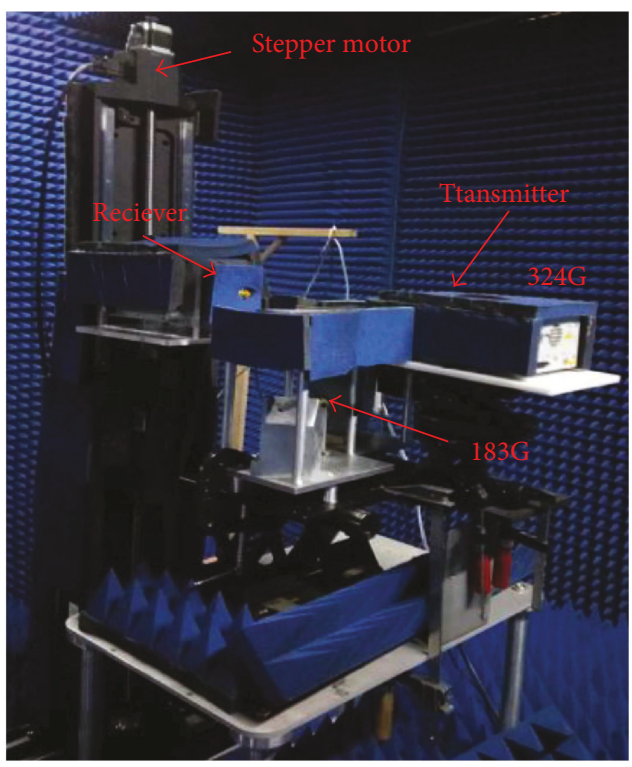

FIGURE 8: The planar near-field measurement of dual-channel 3D QON system.

symmetrical aperture field distribution of the WR3 corrugated feed horn at $324 \mathrm{GHz}$ shown in Figure 3(f). This asymmetry effect mainly comes from the corrugated horn because the consistency energy coefficient $\eta_{s}$ of polarizer is close to 99.96\% relative to the direct transmission through air, which illustrates the correctness of transmission channel. Another interesting fact is that the diffraction from the mirrors' edge and system frames is less than that occurring at the $183 \mathrm{GHz}$ channel obviously, so the amplitude and phase ripple have smaller ranges in the plane.

However, when the filter uses FSS exactly shown in Figure 10(a), the distorted beam appears more serious both in copolarization and cross-polarization amplitude with a beam-expanded peculiarity. Unfortunately, both amplitudes have a slower attenuation and larger beam shape when the amplitude levels are far away from the center of $-10 \mathrm{~dB}$ in the horizontal direction, although both phases have small difference relative to the case of using polarizer and air obviously. This phenomenon results from a worse center flatness of FSS in the horizontal direction due to a small thickness with only $0.4 \mathrm{~mm}$, and meanwhile this perforated array structure has many holes throughout the whole plate surface. On the other hand, with the increasing of insertion loss with $-0.53 \mathrm{~dB}$ at the $324 \mathrm{GHz}$, the transmission result of FSS has the worst isolation in its cross-polarization field, which has larger warped profile range both in the amplitude and in the phase comparing the two others.

In more detail, comparing the testing case of direct air, the polarizer can play a good role in the beam transmission. Obviously, the FSS has caused some problems that are energy loss and directional variation both in the double amplitude, where the energy loss and vector change of copolarization amplitude have reached $3.4 \%$ and $6.3 \%$ relative to the polarizer seen from Table 2 . What is more, the three beam radiuses by simulation and measurement have some difference likewise relative to the theoretical value with $\omega_{\text {out }, 324 \mathrm{G}}=16.35 \lambda_{324 \mathrm{G}}$. The measurement differences reach the range from $1.33 \%$ to $4.61 \%$ due to some test errors mentioned in the $183 \mathrm{GHz}$ channel. What is more, when the beam passes through the filter, the theoretical calculation of beam radius is direct as air. The change of radius does not consider an influence from internal structure, which leads to bring a larger deviation than the direct reflection during the $183 \mathrm{GHz}$ channel. Of course, for most cases of waist position, if the input distance D1 changes $-1 \mathrm{~mm}$ and $+1 \mathrm{~mm}$, the theoretical output radius in the D6 will change $-0.91 \lambda_{324 \mathrm{G}}$ and $+1.147 \lambda_{324 \mathrm{G}}$. Based on such facts, the good flatness and the low insertion loss of filter in the QON system should be considered more to reduce the beam distortion phenomenon especially in the high frequency.

Just like the cross-polarization level, $-29 \mathrm{~dB}$, of double transmitted WR3 feeds, the simulation polarization values of output field are also close to it. However, the measured values have a large difference, where the cross-polarization level using the FSS has only $-13.45 \mathrm{~dB}$ but the polarizer can reach up to $-19.93 \mathrm{~dB}$ that is better than the direct air with $-18.44 \mathrm{~dB}$. The planar cross-polarization level of polarizer also has faster attenuation characteristics than that in the FSS. This phenomenon happened in the case of transmission channel, and it can be understood adequately that the polarizer can isolate another polarization field in the horizontal direction when the polarization of input field has a vertical direction. Furthermore, the cross-polarization using polarizer has even more amplitude plane due to the improvement of polarization purity.

\section{Far-Field Radiation Pattern Results of QON System}

Traditionally, the method to indirectly obtain the 3D far-field radiation pattern is to make full use of the results of near-field planar measurement, where it finishes the near-to-far-field transformation from time domain to frequency spectrum domain by the Fourier transform (FT). In this method, the vector spherical wave spectrum function $f_{t}\left(k_{x}, k_{y}\right)$ is firstly obtained by this transformation based on double tangential E-field complex components $E_{t x}(x, y, z=D 6)$ and $E_{t y}(x, y$, $z=D 6)$ in the $x$ and $y$ direction, respectively, over the bounds of output plane $S_{a}$, and then the far-field radiation pattern $E(r, \theta, \phi)$ is further obtained in terms of spherical spectrum functions $f_{x}(\theta, \phi)$ and $f_{y}(\theta, \phi)$ shown as follows $[32,37]$

$$
\begin{aligned}
\widehat{E}(r, \theta, \phi)= & j k_{0} \frac{\exp \left(-j k_{0} r\right)}{2 \pi r}\left[\widehat{\theta}\left(f_{x} \cos \phi+f_{y} \sin \phi\right)\right. \\
& \left.+\widehat{\phi} \cos \theta\left(-f_{x} \sin \phi+f_{y} \cos \phi\right)\right],
\end{aligned}
$$

where $k_{0}$ is the wavenumber and $k_{x}=k_{0} \sin \theta \cos \phi$ and $k_{y}=k_{0} \sin \theta \sin \phi$ are propagation constant.

The direct far-field pattern measurement in the CATR using an approximate plane wave has become a trend in 

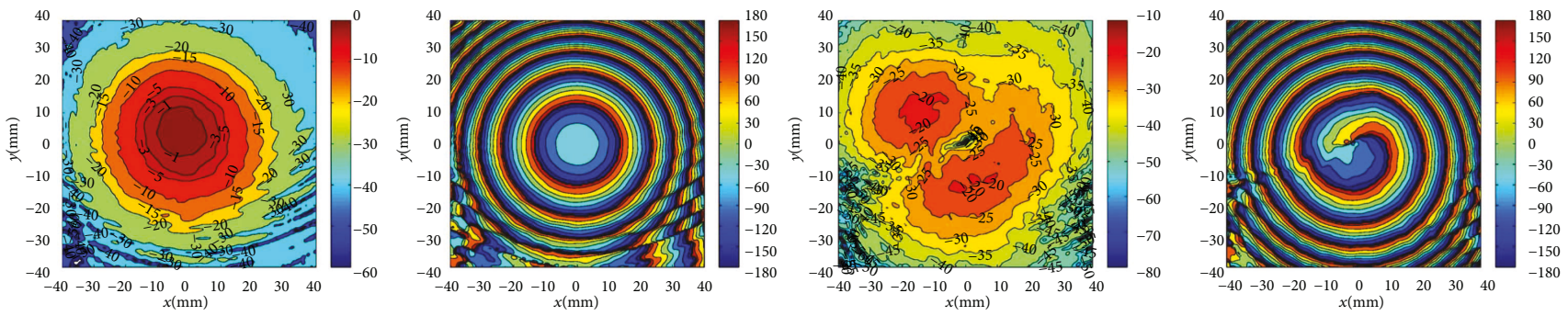

(a) Near-field planar measurement by filter using FSS
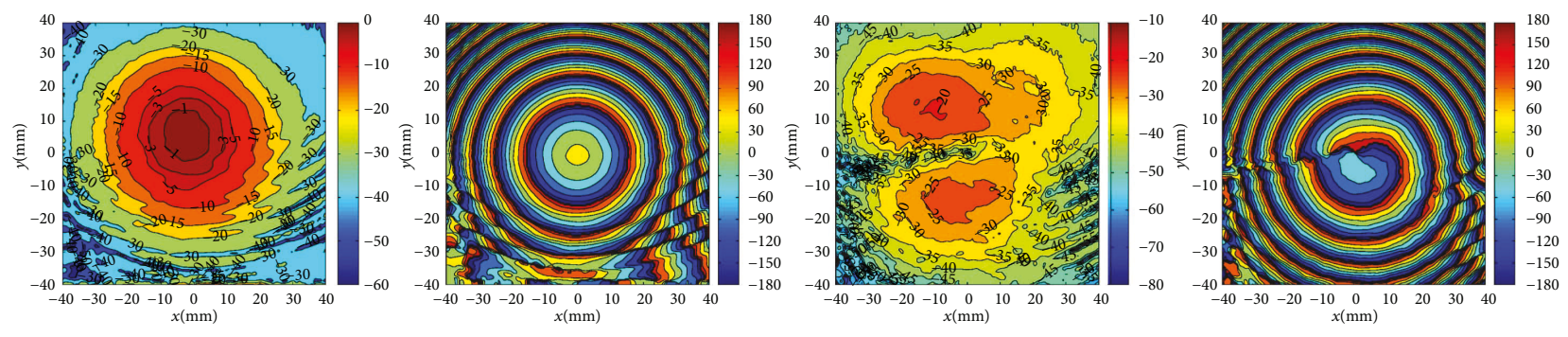

(b) Near-field planar measurement by filter using polarizer
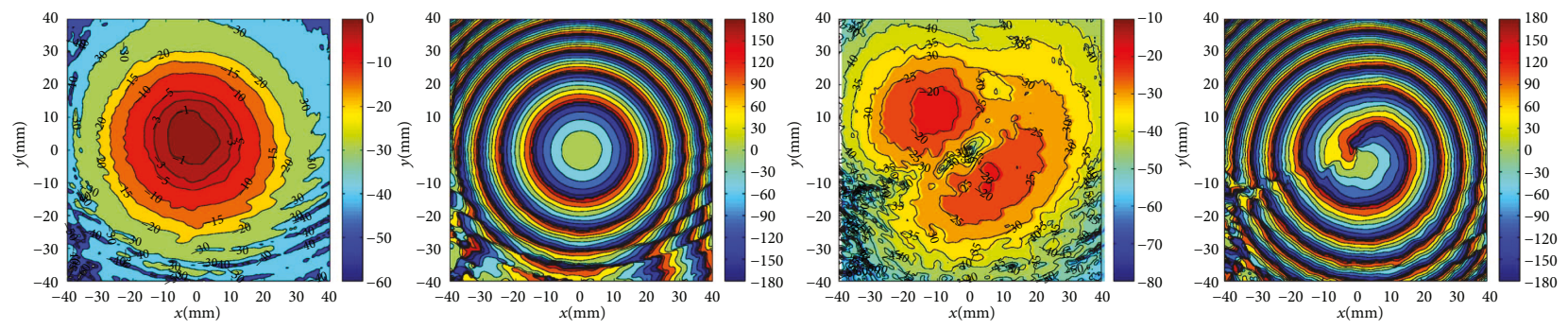

(c) Near-field planar measurement by filter using copper plate
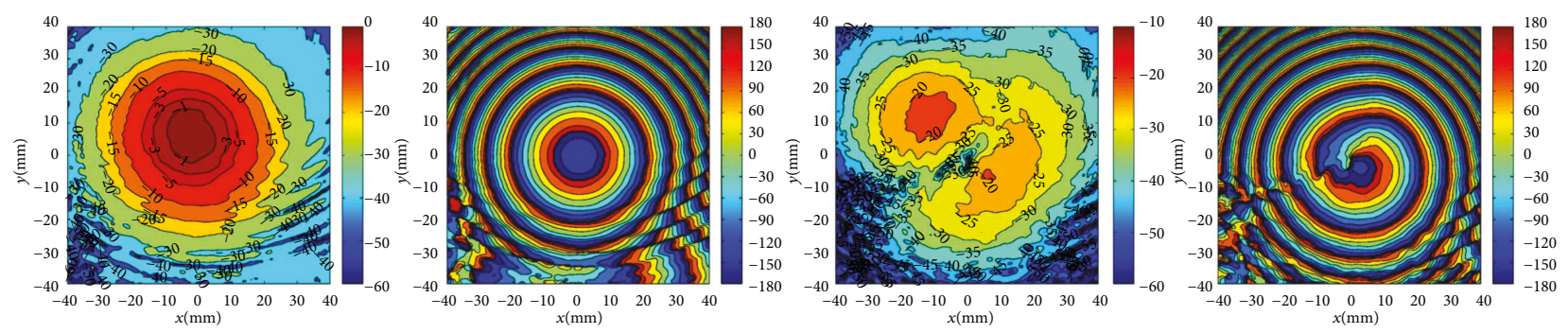

(d) Near-field planar measurement by filter using aluminum plate

FIGURE 9: The near-field measurement results of $183 \mathrm{GHz}$ channel in the dual-channel 3D QON system. From left to right are the measured plane of copolarization amplitude, the copolarization phase, the cross-polarization amplitude, and the cross-polarization phase.

TABLE 1: The near-field measurement data of $183 \mathrm{GHz}$ channel.

\begin{tabular}{|c|c|c|c|c|c|c|c|}
\hline \multirow[t]{2}{*}{ Filter } & \multicolumn{2}{|c|}{ Cross-polarization level } & \multicolumn{3}{|c|}{ Beam radius $(\omega)$ at $D 6=160 \mathrm{~mm}$} & \multicolumn{2}{|c|}{$\begin{array}{l}\text { Measurement } \\
\text { consistency }\end{array}$} \\
\hline & Simulation & Measurement & Simulation & Measurement & Theoretical value & $\eta_{s}$ & $\eta_{v}$ \\
\hline FSS & $-22.05 \mathrm{~dB}$ & $-18.33 \mathrm{~dB}$ & $19.295 \mathrm{~mm}=11.769 \lambda$ & $18.765 \mathrm{~mm}=11.447 \lambda$ & \multirow{4}{*}{$18.152 \mathrm{~mm}=11.07 \lambda$} & $98.9749 \%$ & $97.0155 \%$ \\
\hline Polarizer & $-21.97 \mathrm{~dB}$ & $-18.82 \mathrm{~dB}$ & $18.715 \mathrm{~mm}=11.416 \lambda$ & $18.675 \mathrm{~mm}=11.391 \lambda$ & & $98.7954 \%$ & $97.9952 \%$ \\
\hline Copper plate & $-21.96 \mathrm{~dB}$ & $-18.07 \mathrm{~dB}$ & $18.899 \mathrm{~mm}=11.528 \lambda$ & $19.060 \mathrm{~mm}=11.627 \lambda$ & & $99.0955 \%$ & $97.5476 \%$ \\
\hline Aluminum plate & $-21.96 \mathrm{~dB}$ & $-17.76 \mathrm{~dB}$ & $19.095 \mathrm{~mm}=11.648 \lambda$ & $18.750 \mathrm{~mm}=11.438 \lambda$ & & $100 \%$ & $100 \%$ \\
\hline
\end{tabular}

evaluating the far-field radiation performance. The corresponding principle and test setup for the far-field CATR measurement can be found in $[45,46]$. In this method, the antenna under test (AUT) is fixed on a rotary table in the $\mathrm{QZ}$ range. Then it rotates around the emergent QZ field in the different $\theta$ orientations, the radiation characteristic of 

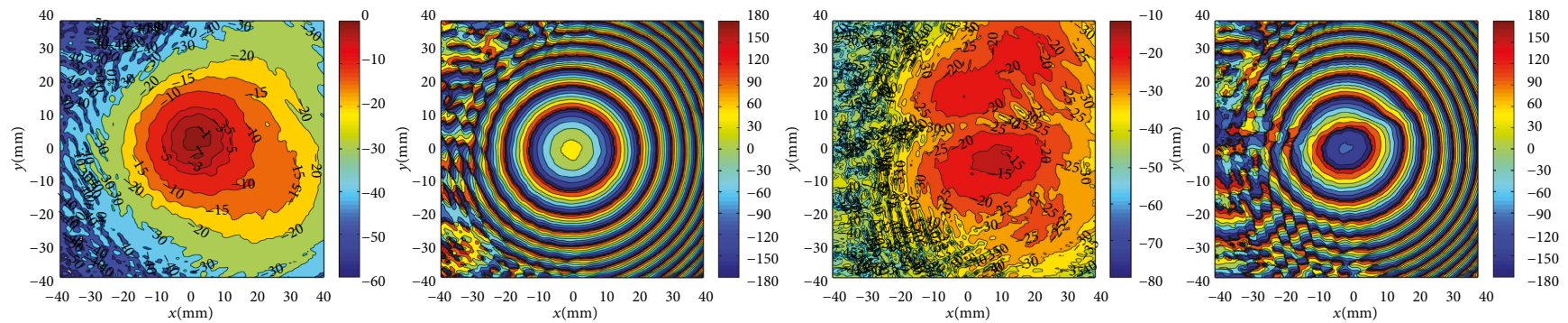

(a) Near-field planar measurement by filter using FSS
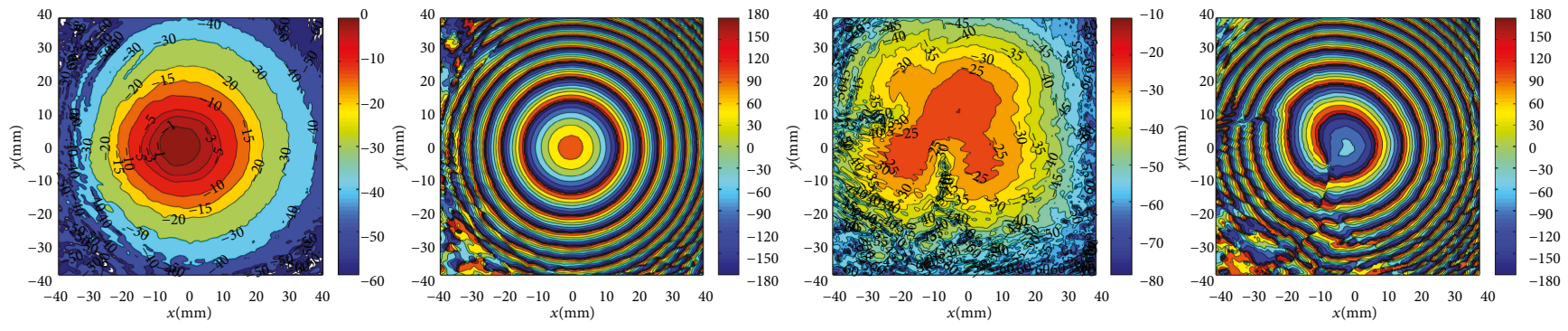

(b) Near-field planar measurement by filter using polarizer
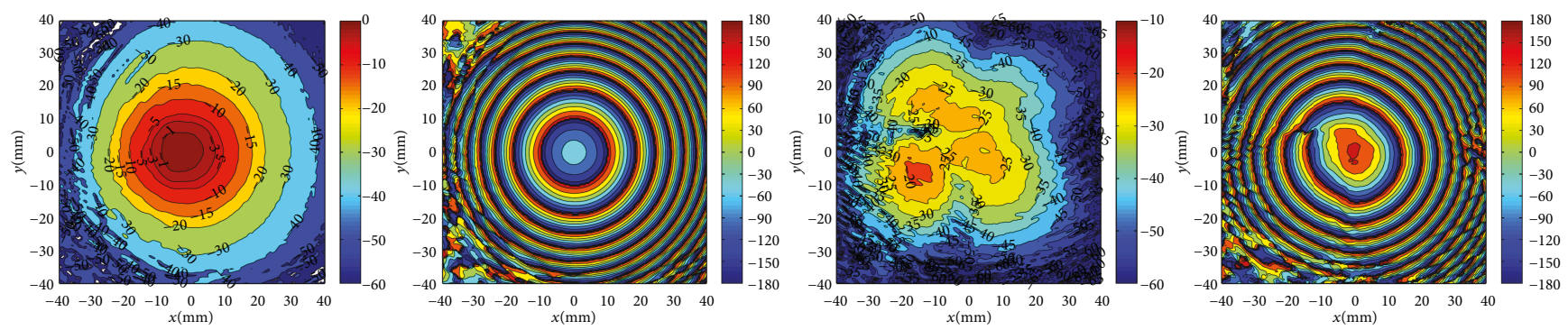

(c) Near-field planar measurement through air

FIGURE 10: The near-field measurement results of $324 \mathrm{GHz}$ channel in the dual-channel 3D QON system. From left to right are the measured plane of copolarization amplitude, the copolarization phase, the cross-polarization amplitude, and the cross-polarization phase.

TABLE 2: The near-field measurement data of $324 \mathrm{GHz}$ channel.

\begin{tabular}{lccccccc}
\hline \multirow{2}{*}{ Filter } & \multicolumn{2}{c}{ Cross-polarization level } & \multicolumn{2}{c}{ Beam radius $(\omega)$ at D6 $=160 \mathrm{~mm}$} & \multicolumn{3}{c}{ Measurement } \\
& Simulation & Measurement & Simulation & Measurement & Theoretical value & $\eta_{s}$ & $\eta_{v}$ \\
\hline FSS & $-29.47 \mathrm{~dB}$ & $-13.45 \mathrm{~dB}$ & $15.710 \mathrm{~mm}=16.966 \lambda$ & $14.44 \mathrm{~mm}=15.595 \lambda$ & & $96.5117 \%$ & $92.3030 \%$ \\
Polarizer & $-32.06 \mathrm{~dB}$ & $-19.93 \mathrm{~dB}$ & $15.375 \mathrm{~mm}=16.605 \lambda$ & $14.74 \mathrm{~mm}=15.919 \lambda$ & $15.145 \mathrm{~mm}=16.35 \lambda$ & $99.9649 \%$ & $98.6356 \%$ \\
Air & $-31.91 \mathrm{~dB}$ & $-18.44 \mathrm{~dB}$ & $15.450 \mathrm{~mm}=16.686 \lambda$ & $14.89 \mathrm{~mm}=16.131 \lambda$ & $100 \%$ & $100 \%$ \\
\hline
\end{tabular}

AUT can be obtained at any direction by recording the received fields in a very short period of time that is shown in Figure 11. The theoretical knowledge comes from the integral between the aperture field distribution $A\left(x_{a}\right)$ of AUT and the QZ field $E_{\mathrm{qz}}$ that can be represented as [33]

$$
F(\theta)=\int A\left(x_{a}\right) E_{\mathrm{qz}}(x, z) d x_{a},
$$

where each section position of $\theta$ direction needs to have an integral over the whole output aperture of AUT in Figure 11. The emergent direction of QZ field comes from negative $z$-axis, and the aperture of AUT is rotated in the
XOZ plane. $E_{\mathrm{qz}}(x, z)=E_{\mathrm{qz}}\left(x_{a} \cos \theta,-x_{a} \sin \theta\right)=a_{0}\left(x_{a} \cos \theta\right.$, $\left.-x_{a} \sin \theta\right) \exp \left(j \gamma_{0}\right) \exp \left(-j k_{0} z\right)$ is the incident QZ field on the AUT with a $\theta$ orientation. $a_{0}(x, z)=1$ and $\gamma_{0}=0$ are the amplitude and phase of the ideal planar wavefront.

In the actual measurement environment, there are still some stray signals from the environments, such as the diffraction phenomenon from the testing system structures, the influence of mirror surface error from larger reflectors in creating the QZ field, and the random noise in measurement equipment, which can bring the ripple of amplitude and phase in the QZ field, where this typically predicted amplitude ripple is within $\pm 0.5 \mathrm{~dB}$, and phase ripple is within $\pm 5^{\circ}[30,39]$. Those influences from the quality of 


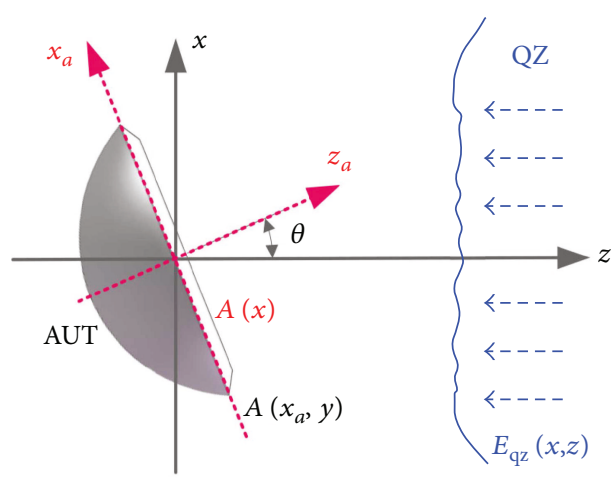

FIgURE 11: The far-field pattern measurement in the CATR.

QZ in the tri-CATR are considered below by comparing with ideal QZ integration.

Due to the fact that far-field pattern testing in the CATR needs double same corrugated feed horns in the transmitter and receiver each other, the far-field of $183 \mathrm{GHz}$ channel is merely indirectly obtained based on the near-field measurement and the near-to-far-field transformation for only using one WR4 corrugated horn, however, the $324 \mathrm{GHz}$ channel is directly measured in the tri-CATR by double WR3 corrugated horns, which is implemented from the range $-90 \mathrm{deg}$ to $90 \mathrm{deg}$ within a sampling interval of $0.1 \mathrm{deg}$, and then those results are compared by the same aperture integral method using ideal plane wave. The simulation results of far-field are also similarly obtained by the PO current method in GRASP. The different far-field results in such double channels are also discussed and compared below.

4.1. The $183 \mathrm{GHz}$ Far-Field Results. The four planar far-field amplitudes by near-to-far transformation are shown in Figure 12(a) based on each near-field test results. The cut section results of the FSS and the polarizer are also compared with amplitude and phase in Figures 12(b) and 12(c), where the simulation, near-to-far-field transformation based on the near-flied measurement, and the aperture integral using ideal QZ field are compared and summed up in Table 3.

Benefiting from the near-field planar test with a feature of circular symmetry Gaussian distribution, the far-field copolarization planar amplitude by near-to-far-field transformation is also consistent both in the $\theta$ and in the $\phi$ direction, so the four radiation patterns have small difference in the copolarization amplitude shown in Figure 12(a), where the three scalar and vector coefficients of planar far-field are also calculated relative to the results of aluminum plate that have $\eta_{s, \mathrm{FSS}}=99.5457 \%$ and $\eta_{v, \mathrm{FSS}}=98.7896 \%$ for FSS, $\eta_{s \text {, polari- }}$ zer $=99.9157 \%$ and $\eta_{v \text {, polarizer }}=99.2113 \%$ for polarizer, and $\eta_{s, \text { copper }}=99.5442 \%$ and $\eta_{v \text {, copper }}=98.8688 \%$ for copper plate. Those transformation efficiencies are obviously superior to that in the near-field measurement shown in Table 1, which mainly benefits from the rapid attenuation in far-field.

Comparing with the first side-lobe level of copolarization amplitude both in phi $=0 \mathrm{deg}$ and in $\mathrm{phi}=90 \mathrm{deg}$ from Figures 12(b) and 12(c) and summarized in Table 3, the calculation values by the near-to-far-field transformation have $-40 \mathrm{~dB}$ to $-32 \mathrm{~dB}$, which is generally higher $10 \mathrm{~dB} \sim 20 \mathrm{~dB}$ than the simulation values but close to the aperture integrating values. Both differences with respect to the simulation come from the stray signals in testing environment and are limited by the side-lobe level of transmitted WR4 horn with only $-39 \mathrm{~dB}$. It is also obvious that the first side-lobe level cannot be significantly decreased by the ideal QZ field integral for relying on the test condition of near-field. The cut section of copolarization phase has the same shape among the three far-field results, but the phase of simulation has a difference with $2 \pi$ comparing with the two others.

The cross-polarization level in the simulation is close to the case of near-to-far-field transformation. The isolation effect of the aluminum plate is slightly superior to the copper plate, but the polarizer has the best isolation effect than three other results both in phi $=0 \mathrm{deg}$ and in $\mathrm{phi}=90 \mathrm{deg}$, where the cross-polarization level reaches $-19.74 \mathrm{~dB}$ and $-30.03 \mathrm{~dB}$, respectively, by the near-to-far-field transformation. When calculated by the aperture integrating, the far-field in the double directions is achieved using the same cut section field based on near-field measurement. Due to the QZ field being an ideal plane wave in the aperture integrating, the cross-polarization level can be changed both in the phi $=0$ deg and in phi $=90 \mathrm{deg}$. The biggest change happens when the filter uses polarizer, so the quality of QZ field can influence the cross-polarization level of radiation far-field; however, it is difficult to forecast whether it is good or not because there are some randomness amplitude and phase ripples in the actual QZ field when the AUT is measured at the different positions.

4.2. The $324 \mathrm{GHz}$ Far-Field Results. The three far-field planar amplitudes of copolarization are similarly obtained by a near-to-far-field transformation and shown in Figure 13(a). Just like an influence in the near-field test, the far-field pattern using the FSS has obvious pattern distortion and asymmetry especially in the section direction near phi $=0^{\circ}$, where the coefficients of far-field planar pattern have $\eta_{s, \mathrm{FSS}}=98.7915 \%$ and $\eta_{v \text {,FSS }}=96.9797 \%$ relative to air. However, the far-field planar pattern of polarizer has a very good consistent circular symmetry feature both in the $\theta$ and in the $\phi$ direction relative to the air, where it has the coefficients of $\eta_{s \text {, polarizer }}=99.9887 \%$ and $\eta_{v \text {, polarizer }}=99.5524 \%$ that can verify the polarizer has a better transmission performance at the $324 \mathrm{GHz}$. Of course, the planar far-field has bigger noncircular symmetry Gaussian distribution in the phi $=90^{\circ}$ due to the asymmetric aperture field of the WR3 horn itself.

The cut section results of far-field patterns using the FSS and the polarizer are also presented in Figures 13(b) and 13(c), where the simulation, near-to-far-field transformation, and the aperture integral values using ideal QZ are given. What is more, the horizontal cut section of far-field pattern has been obtained at $324 \mathrm{GHz}$ by the direct far-field measurement by the tri-reflector CATR system in the BUPT [29]. Their far-field results of amplitude and phase have been shown in Figure 13(d). Those relevant results are also compared and summed up in Table 4.

Comparing with the side-lobe level of copolarization amplitude, the near-to-far-field transformation can reach $-45 \mathrm{~dB} \sim-39 \mathrm{~dB}$, which are general higher $10 \mathrm{~dB} \sim 20 \mathrm{~dB}$ than 

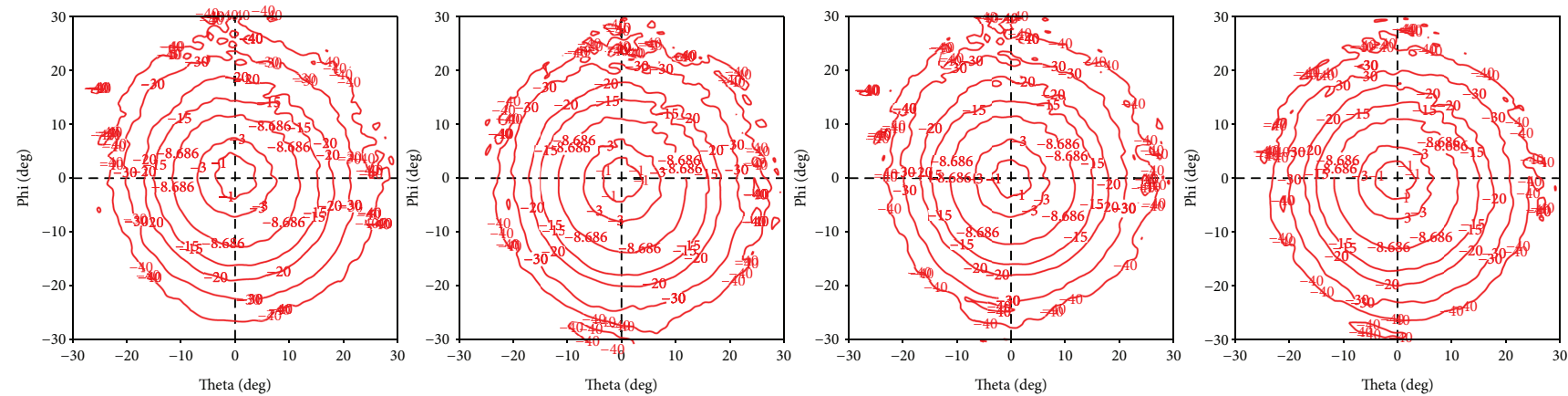

(a) The far-field planar amplitude by near-to-far-field transformation. From left to right are FSS, polarizer, copper, and aluminum

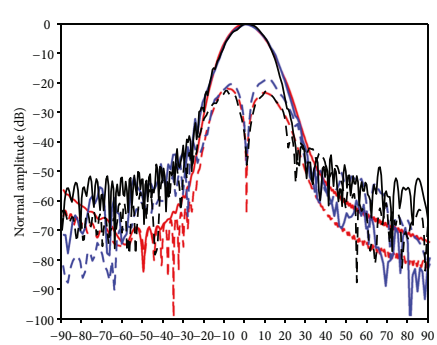

Theta (deg)

Simulation $\mathrm{Co}$ Phi $=0 \quad \ldots-\ldots$ NF to FF Cx Phi $=0$

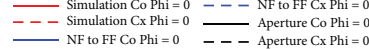

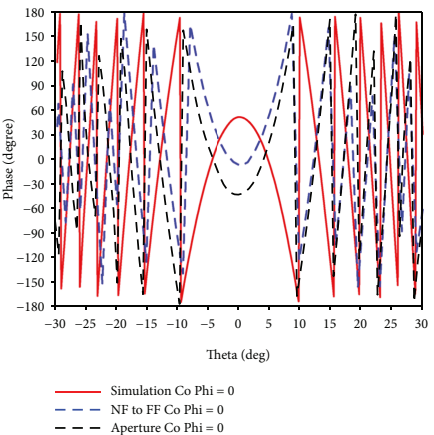

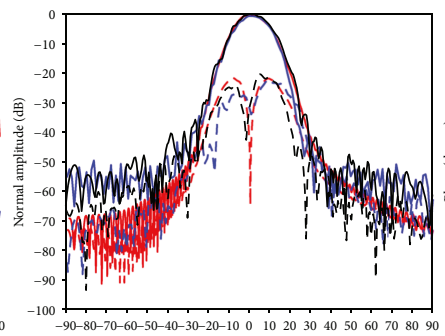

Theta (deg)

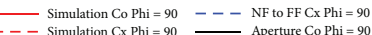

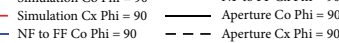

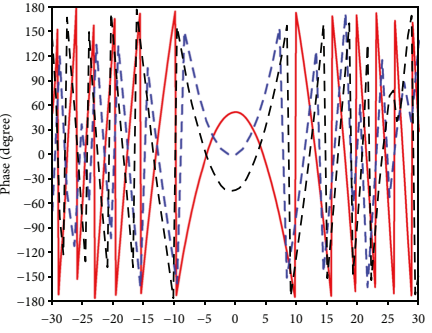

Theta (deg)

Simulation $\mathrm{Co}$ Phi $=0$

(b) FSS: the amplitude and phase of far-field by simulation, near-to-far-field transformation, and aperture field integral

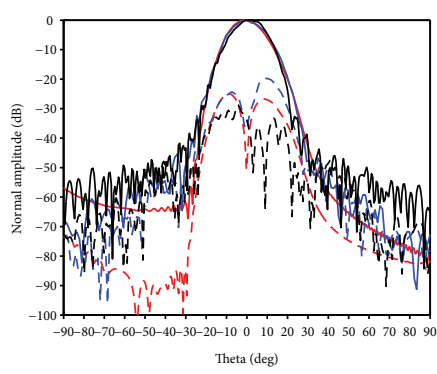

Theta (deg)

Simulation $\mathrm{Co} P$ Phi $=0 \quad---\mathrm{NF}$ to $\mathrm{FF} \mathrm{Cx}$ Phi $=0$

$\begin{array}{lll}-- & \text { Simulation } \mathrm{Cx} \text { Phi }=0 \\ \text { NF to FF Co Phi }=0 & \text { Aperture } \mathrm{Co} \text { Phi }=0 \\ --- \text { Aperture } \mathrm{Cx} \text { Phi }=0\end{array}$

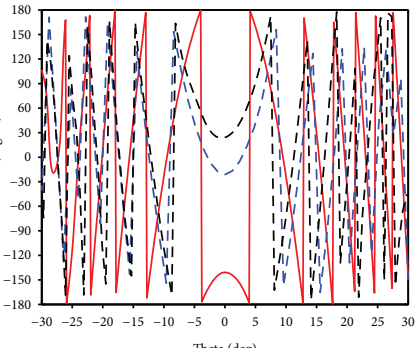

Theta (deg)

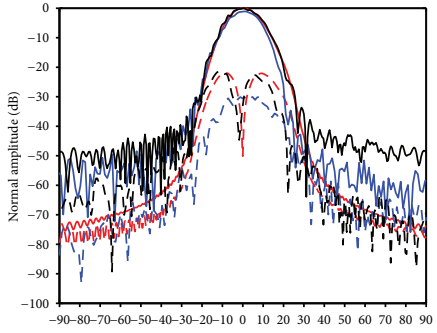

Theta (deg)

-- Simulation $\mathrm{Co}$ Phi $=0$

$-\mathrm{NF}$ to $\mathrm{FF} \mathrm{CO} \mathrm{Phi}=0$

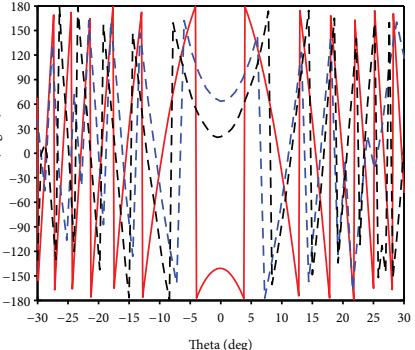

Theta $(\mathrm{deg})$

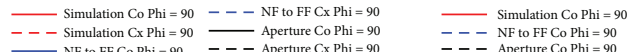

(c) Polarizer: the amplitude and phase of far-field by simulation, near-to-far-field transformation, and aperture field integral

FIgURE 12: The far-field radiation pattern results of $183 \mathrm{GHz}$ channel.

TABLE 3: The far-field results of $183 \mathrm{GHz}$ channel.

\begin{tabular}{|c|c|c|c|c|c|c|c|}
\hline \multirow{2}{*}{ Filter } & \multirow{2}{*}{ Angle (degree) } & \multicolumn{3}{|c|}{ Cross-polarization level } & \multicolumn{3}{|c|}{ First side-lobe of copolarization amplitude } \\
\hline & & Simulation & Near-to-far & Ideal QZ integral & Simulation & Near-to-far & Ideal QZ integral \\
\hline \multirow{2}{*}{ FSS } & $\mathrm{Phi}=0$ & $-22.26 \mathrm{~dB}$ & $-18.71 \mathrm{~dB}$ & $-22.83 \mathrm{~dB}$ & $-55.27 \mathrm{~dB}$ & $-32.48 \mathrm{~dB}$ & $-33.56 \mathrm{~dB}$ \\
\hline & Phi $=90$ & $-21.87 \mathrm{~dB}$ & $-22.47 \mathrm{~dB}$ & $-20.58 \mathrm{~dB}$ & $-45.75 \mathrm{~dB}$ & $-34.85 \mathrm{~dB}$ & $-34.32 \mathrm{~dB}$ \\
\hline \multirow{2}{*}{ Polarizer } & $\mathrm{Phi}=0$ & $-25.09 \mathrm{~dB}$ & $-19.74 \mathrm{~dB}$ & $-29.53 \mathrm{~dB}$ & $-56.75 \mathrm{~dB}$ & $-32.38 \mathrm{~dB}$ & $-32.71 \mathrm{~dB}$ \\
\hline & Phi $=90$ & $-22.09 \mathrm{~dB}$ & $-30.03 \mathrm{~dB}$ & $-21.34 \mathrm{~dB}$ & $-55.42 \mathrm{~dB}$ & $-34.67 \mathrm{~dB}$ & $-32.36 \mathrm{~dB}$ \\
\hline \multirow{2}{*}{ Copper plate } & Phi $=0$ & $-21.89 \mathrm{~dB}$ & $-17.96 \mathrm{~dB}$ & $-22.32 \mathrm{~dB}$ & $-60.19 \mathrm{~dB}$ & $-40.37 \mathrm{~dB}$ & $-41.8 \mathrm{~dB}$ \\
\hline & Phi $=90$ & $-22.12 \mathrm{~dB}$ & $-20.57 \mathrm{~dB}$ & $-21.47 \mathrm{~dB}$ & $-50.19 \mathrm{~dB}$ & $-40.15 \mathrm{~dB}$ & $-35.2 \mathrm{~dB}$ \\
\hline \multirow{2}{*}{ Aluminum plate } & $\mathrm{Phi}=0$ & $-21.89 \mathrm{~dB}$ & $-18.39 \mathrm{~dB}$ & $-22.98 \mathrm{~dB}$ & $-62.11 \mathrm{~dB}$ & $-35.94 \mathrm{~dB}$ & $-30.88 \mathrm{~dB}$ \\
\hline & $\mathrm{Phi}=90$ & $-21.93 \mathrm{~dB}$ & $-21.87 \mathrm{~dB}$ & $-22.07 \mathrm{~dB}$ & $-51.65 \mathrm{~dB}$ & $-36.76 \mathrm{~dB}$ & $-36.84 \mathrm{~dB}$ \\
\hline
\end{tabular}

the corresponding simulation values because it has also been restricted by the transmitted WR3 horn with a side-lobe level of $-42.3 \mathrm{~dB}$. The best result appears when it uses polarizer with a maximum value $-48.66 \mathrm{~dB}$; however, the FSS has only $-39.08 \mathrm{~dB}$. In the calculation of far-field using ideal QZ field, the side-lobe level becomes worse. Similarly, the far-field by 

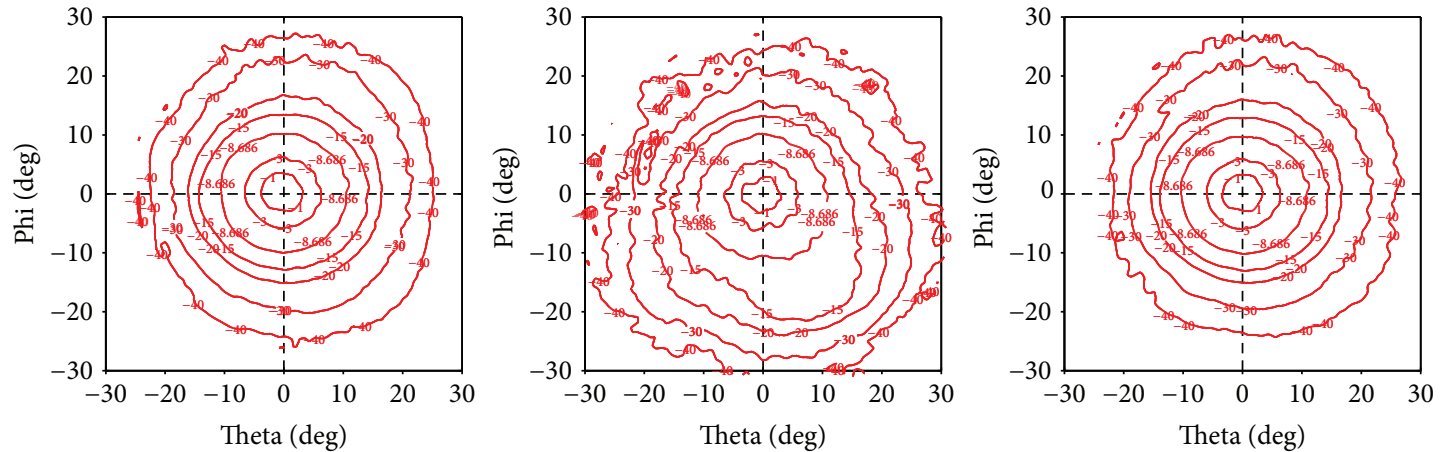

(a) Far-field planar amplitude by near-to-far-field transformation. From left to right are air, FSS, polarizer

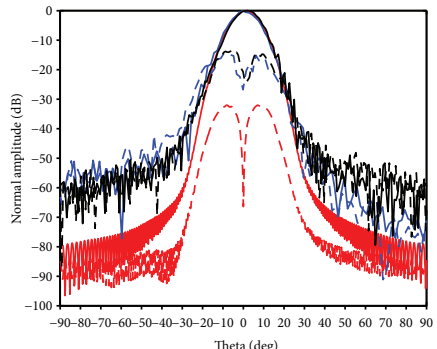

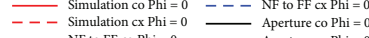

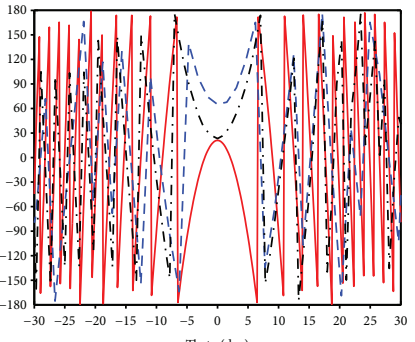

Theta (deg)

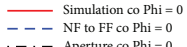

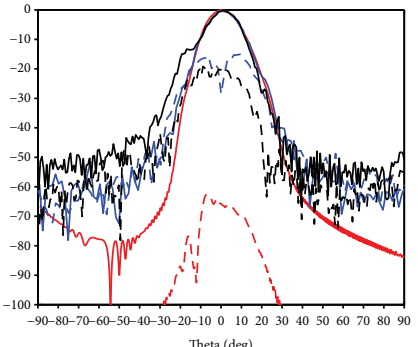

Theta (deg)

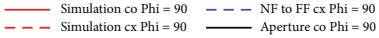

Aperture co Phi $=90$

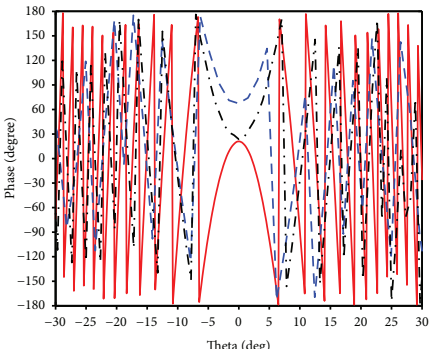

- Simulation co Phi $=90$

(b) FSS: the amplitude and phase of far-field by simulation, near-to-far-field transformation, and aperture field integral

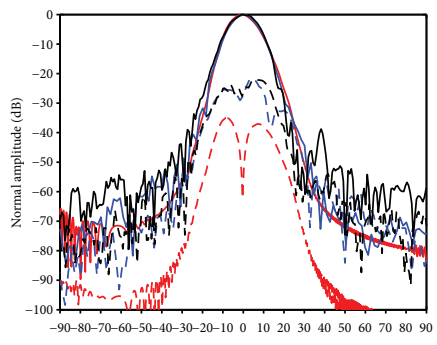

Theta (deg)

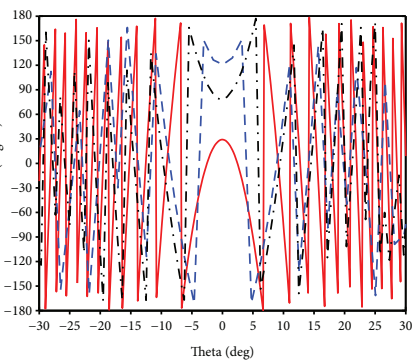

$\begin{array}{ll}\text { Simulation co Phi }=0 \\ --- & \text { NF to } F F \text { co Phi }=0\end{array}$

. - . - Aperture co Phi $=0$

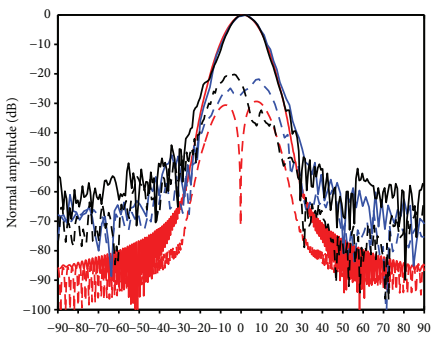

Theta (deg)

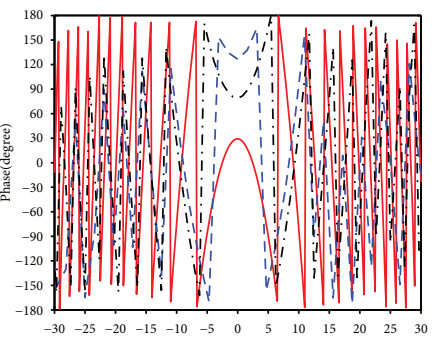

Theta (deg)

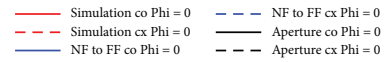

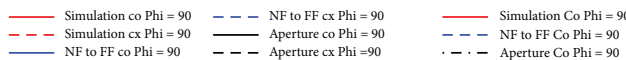

(c) Polarizer: the amplitude and phase of far-field by simulation, near-to-far-field transformation, and aperture field integral
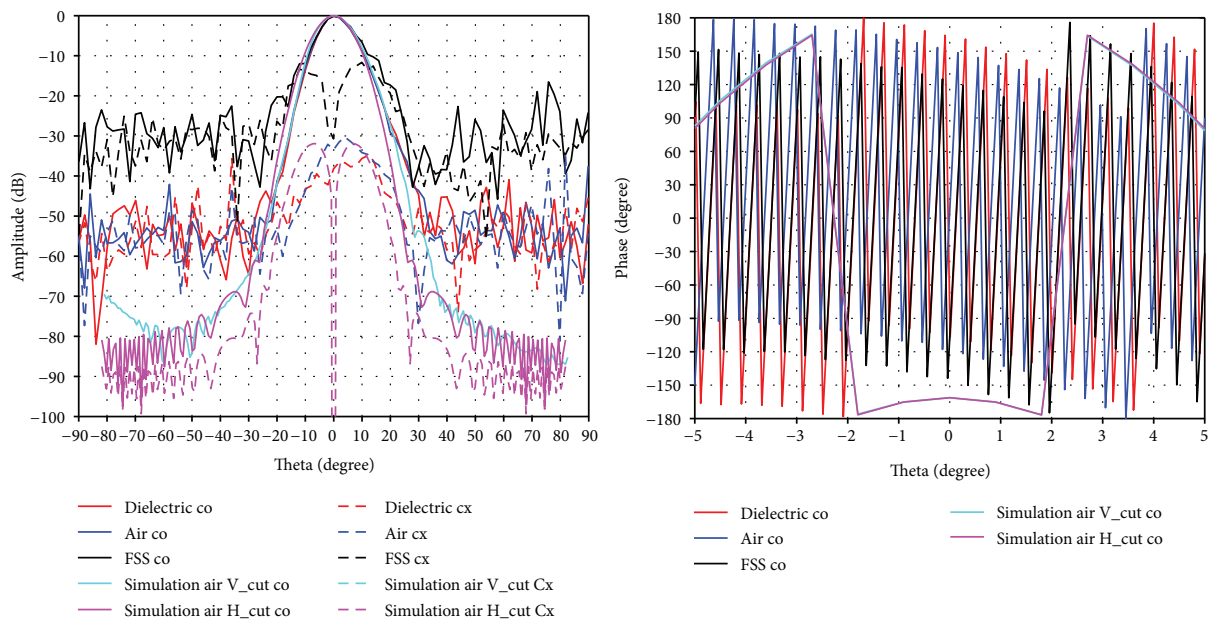

(d) The direct measurement results of far-field radiation pattern in the tri-CATR. The left is amplitude and the right is phase

FIGURE 13: The far-field radiation pattern results of $324 \mathrm{GHz}$ channel. 
TABLE 4: The far-field results of $324 \mathrm{GHz}$ channel.

\begin{tabular}{|c|c|c|c|c|c|c|c|c|c|}
\hline \multirow{3}{*}{ Filter } & \multirow{3}{*}{ Angle (degree) } & \multirow[b]{3}{*}{ Simulation } & \multicolumn{3}{|c|}{ Cross-polarization level } & \multicolumn{4}{|c|}{ First side-lobe of copolarization amplitude } \\
\hline & & & & Far-fiel & d in CATR & & & Far-fie & $\mathrm{d}$ in CATR \\
\hline & & & Near-to-far & $\begin{array}{c}\text { Ideal QZ } \\
\text { integral }\end{array}$ & Measurement & Simulation & Near-to-far & $\begin{array}{c}\text { Ideal QZ } \\
\text { integral }\end{array}$ & Measurement \\
\hline \multirow{2}{*}{ FSS } & $\mathrm{Phi}=0$ & $-32.01 \mathrm{~dB}$ & $-14.44 \mathrm{~dB}$ & $-16.78 \mathrm{~dB}$ & $-11.64 \mathrm{~dB}$ & $-53.08 \mathrm{~dB}$ & $-39.08 \mathrm{~dB}$ & $-27.09 \mathrm{~dB}$ & $-22.67 \mathrm{~dB}$ \\
\hline & $\mathrm{Phi}=90$ & $-62.66 \mathrm{~dB}$ & $-15.04 \mathrm{~dB}$ & $-15.53 \mathrm{~dB}$ & - & $-58.96 \mathrm{~dB}$ & $-38.86 \mathrm{~dB}$ & $-32.32 \mathrm{~dB}$ & - \\
\hline \multirow{2}{*}{ Polarizer } & $\mathrm{Phi}=0$ & $-35.04 \mathrm{~dB}$ & $-21.94 \mathrm{~dB}$ & $-21.85 \mathrm{~dB}$ & $-34.93 \mathrm{~dB}$ & $-64.17 \mathrm{~dB}$ & $-48.66 \mathrm{~dB}$ & $-38.69 \mathrm{~dB}$ & $-45.22 \mathrm{~dB}$ \\
\hline & $\mathrm{Phi}=90$ & $-30.61 \mathrm{~dB}$ & $-21.77 \mathrm{~dB}$ & $-19.76 \mathrm{~dB}$ & - & $-68.79 \mathrm{~dB}$ & $-44.43 \mathrm{~dB}$ & $-36.89 \mathrm{~dB}$ & - \\
\hline \multirow{2}{*}{ Air } & $\mathrm{Phi}=0$ & $-31.89 \mathrm{~dB}$ & $-24.38 \mathrm{~dB}$ & $-21.99 \mathrm{~dB}$ & $-30.3 \mathrm{~dB}$ & $-53.78 \mathrm{~dB}$ & $-40.49 \mathrm{~dB}$ & $-39.05 \mathrm{~dB}$ & $-46.57 \mathrm{~dB}$ \\
\hline & $\mathrm{Phi}=90$ & $-295.6 \mathrm{~dB}$ & $-22.24 \mathrm{~dB}$ & $-26.14 \mathrm{~dB}$ & - & $-60.1 \mathrm{~dB}$ & $-45.46 \mathrm{~dB}$ & $-41.4 \mathrm{~dB}$ & - \\
\hline
\end{tabular}

the ideal aperture integral based on the near-field testing results cannot make the side-lobe level of copolarization amplitude better.

When the $324 \mathrm{GHz}$ channel is directly measured in the tri-CATR, their side-lobe levels have an obvious difference. This variation has been fully shown in Figure 13(d). It is the case of without any filter that has the best side-lobe level of $-46.57 \mathrm{~dB}$, which has thoroughly closed to the ideal simulation situation. When the filter adopts the polarizer, the effect in the side-lobe becomes excellent as low as up to $-45.22 \mathrm{~dB}$, so the polarizer has the same transformation characteristics just like through the air. Of course, the FSS has also the worst side-lobe level for only $-22.67 \mathrm{~dB}$. Meanwhile, the tested copolarization phase has the same shape with periodic-serrated oscillation function, where it has a periodic $2 \pi$ change with the angle $\theta$ that are different from the simulation with a Gaussian distribution characteristic in the main-lobe range.

Although the far-field calculation by near-to-far and aperture integral are similar both in cross-polarization levels, their test in the CATR still has a large variation. From the cut section results, the terrible cross-polarization level still happens in the FSS for only $-11.64 \mathrm{~dB}$ that is lower near $-19 \mathrm{~dB}$ relative to the case of direct air. The good phenomenon similarly appears on the polarizer, where the crosspolarization level can reach up to $-34.93 \mathrm{~dB}$ that has a similar and better isolation than that through the air with $-30.3 \mathrm{~dB}$. Based on those facts, it can be derived that the direct test of far-field radiation pattern in the CATR still has an important and indispensable role in evaluating the QON system performance especially in the cross-polarization and side-lobe levels just like the simulation.

\section{Discussion and Conclusion}

A dual-channel 3D QON system has been analyzed and compared using polarizer and FSS that transmits the $324 \mathrm{GHz}$ signal on the top layer while diverting the $183 \mathrm{GHz}$ signal to the bottom layer. The changes of beam radiuses are firstly traced in such system by the complex beam parameter and system transfer matrix for deciding the size of mirrors and calculating the beam distortion. Of course, the quality of corrugated feed horn can influence the radiation feature of output field, so the mode components of double corrugated feed horns are calculated over the horn aperture by the SWE method, which is used to explain the reason of measurement with asymmetry pattern in the near-field and far-field results in $324 \mathrm{GHz}$ channel. What is more, the design principle of FSS with perforated hexagonal array and WGP printed on the dielectric substrate is also discussed and verified by testing transmission coefficients.

In the planar near-field measurement, the $183 \mathrm{GHz}$ channel is reflected at four different cases with FSS, polarizer, copper, or aluminum plate. Meanwhile, the $324 \mathrm{GHz}$ channel is transmitted through FSS, polarizer, or direct air. Generally, the beam radiuses in both channels can agree very well in the all measurement results, but the difference with the theoretical values is small and depends on the actual measurement environment conditions. In the $183 \mathrm{GHz}$ channel, comparing the planar copolarization and cross-polarization results, the four different reflectors can achieve very good reflection results both in the amplitude and phase. Their symmetry of planar patterns, cross-polarization level, and side-lobe levels is very similar. However, in the $324 \mathrm{GHz}$ channel, the cross-polarization level and side-lobe level using the polarizer have the best performance. What is more, the symmetry of planar near-field using FSS further decreases when the beam is transmitted.

The far-field patterns of $183 \mathrm{GHz}$ are indirectly obtained based on different methods. The near-to-far-field transformation and aperture integral using ideal plane wave depend on the measurement condition of near-field. Their planar and cut section of far-fields are solved out and discussed both in the phi $=0 \mathrm{deg}$ and in the $\mathrm{phi}=90 \mathrm{deg}$, which have similar results just like the near-field. Meanwhile, the direct far-field measurement of $324 \mathrm{GHz}$ is also implemented by the trireflectors CATR with an anechoic chamber in the BUPT. In this case, the cross-polarization level and the side-lobe level are more outstanding. Although the near-field measurement in the QON system is time-consuming for satisfying the Nyquist sampling ratio, it can foresee the working performance within whole output plane especially on the symmetry of planar pattern. However, it is also often limited by many test conditions, such as the stray signals, the position drift from scanning frame, and the diffraction phenomena. In the far-field radiation pattern, the aperture integral using ideal QZ field can obtain equivalent cross-polarization level relative to the near-to-far-field transformation, but it has no very good improvement in the side-lobe level for relying on the near-field results. Comparing with such double far-field 
methods, the other method of direct far-field measurement in the CATR can play more important and indispensable role in evaluating the pattern performance especially in the crosspolarization and side-lobe levels. The direct far-field measurement in the CATR is close to the simulation seeing from $324 \mathrm{GHz}$ channel.

Finally, the total experimental results reveal that the polarizer with the dielectric-slab has a better performance both in the reflection and in the transmission for having a symmetrical pattern, high cross-polarization isolation, low energy loss, and side-lobe level. For the FSS with many small periodic holes, when the working frequency increases, the thickness of filter tends to be very thin, so the flatness of filter installation is another important factor to be considered for keeping the symmetry of output field. If the problem cannot be solved correctly, the output beam has the phenomenon of distortion and deformation in the transmitted wave. This distortion of beams and energy loss often happened when the output beam cannot be placed in the focus of next cascade mirrors. The main solution is to make the equivalent focal length of mirrors larger and have a small incident angle. Meanwhile, it requires that the beam radius becomes smaller in the limited space for reducing the diffraction effect. What is more, it should also make sure the reflector mirrors to meet a condition that the beam radius is less than a quarter of the mirror diameter for reducing beam spillover and diffraction.

\section{Conflicts of Interest}

The authors declare that they do not have any commercial or associative interest that represents competing interests in connection with the work submitted.

\section{Acknowledgments}

This work is supported by the National Natural Science Foundation of China under the Contract no. 61401031 and the Scientific Research Foundation for the Returned Overseas Chinese Scholars, State Education Ministry. Furthermore, the authors are grateful to Professor Junsheng Yu for the long-term support and help during the learning and testing process of quasi-optical network system in the BUPT.

\section{References}

[1] P. H. Siegel, “THz instruments for space," IEEE Transactions on Antennas and Propagation, vol. 55, no. 11, pp. 2957-2965, 2007.

[2] R. Jorgensen, G. Padovan, P. De Maagt, and D. Lamarre, “A 5frequency millimeter wave antenna for a spaceborne limb sounding instrument," IEEE Transactions on Antennas and Propagation, vol. 49, no. 5, pp. 703-714, 2001.

[3] C. Dong, J. Yang, Z. Yang et al., "An overview of a new Chinese weather satellite FY-3A," Bulletin of the American Meteorological Society, vol. 90, no. 10, pp. 1531-1544, 2009.

[4] K. Chen, S. English, N. Bormann, and J. Zhu, "Assessment of FY-3A and FY-3B MWHS observations," Weather and Forecasting, vol. 30, no. 5, pp. 1280-1290, 2015.
[5] A. Gonzalez, Y. Uzawa, Y. Fujii, and K. Kaneko, "ALMA band 10 tertiary optics," Infrared Physics and Technology, vol. 54, no. 6, pp. 488-496, 2011.

[6] M. Oldfield, B. P. Moyna, E. Allouis et al., "MARSCHALS: development of an airborne millimeter-wave limb sounder," Proceedings of the International Society for Optical Engineering (SPIE), vol. 4540, 2001.

[7] P. Goldsmith, Quasioptical Systems: Gaussian Beam Quasioptical Propogation and Applications, IEEE Press, 1998.

[8] A. Gonzalez, "Frequency independent design of quasi-optical systems," Journal of Infrared Millimeter and Terahertz Waves, vol. 37, no. 2, pp. 147-159, 2016.

[9] M. Klein and A. J. Gasiewski, "Nadir sensitivity of passive millimeter and submillimeter wave channels to clear air temperature and water vapor variations," Journal of Geophysical Research Atmospheres, vol. 105, no. D13, pp. 17481-17511, 2000.

[10] H. J. Liebe, G. A. Hufford, and M. G. Cotton, "Propagation modeling of moist air and suspended water ice particles at frequencies below 1000 GHz," Atmospheric Propagation Effects Through Natural and Manmade Obscurants for Visible To Mm-Wave Radiation, 1993.

[11] N. R. Council, Handbook of Frequency Allocations and Spectrum Protection for Scientific Uses, National Academy of Sciences, 2007.

[12] J. Miao, T. Rose, K. Kunzi, and P. Zimmermann, "A future millimeter/sub-millimeter radiometer for satellite observation of ice clouds," International Journal of Infrared and Millimeter Waves, vol. 23, no. 8, pp. 1159-1170, 2002.

[13] R. J. Martin and D. H. Martin, "Quasi-optical antennas for radiometric remote-sensing," Electronics \& Communication Engineering Journal, vol. 8, no. 1, pp. 37-48, 1996.

[14] J. E. Mckay, D. A. Robertson, P. A. S. Cruickshank et al., "Compact wideband corrugated feedhorns with ultra-low sidelobes for very high performance antennas and quasi-optical systems," IEEE Transactions on Antennas and Propagation, vol. 61, no. 4, pp. 1714-1721, 2013.

[15] C. Granet and G. L. James, "Design of corrugated horns: a primer," IEEE Antennas and Propagation Magazine, vol. 47, no. 2, pp. 76-84, 2005.

[16] T. Salimi, A. Maghoul, and A. A. Abbasid, "Design of a compact Gaussian profiled corrugated horn antenna for low sidelobe-level applications," International Journal of Computer Theory and Engineering, vol. 5, no. 2, pp. 223-226, 2013.

[17] A. Densmore, Y. Rahmat-Samii, and G. Seck, "Corrugatedconical horn analysis using aperture field with quadratic phase," IEEE Transactions on Antennas and Propagation, vol. 59, no. 9, pp. 3453-3457, 2011.

[18] Y. Beniguel, A. Berthon, C. V. Klooster, and L. Costes, "Design realization and measurements of a high performance wideband corrugated horn," IEEE Transactions on Antennas and Propagation, vol. 53, no. 11, pp. 3540-3546, 2005.

[19] B. A. Munk, Frequency Selective Surfaces: Theory and Design, Wiley, New York, 2005.

[20] R. Dickie, R. Cahill, V. Fusco, H. S. Gamble, and N. Mitchell, "Thz frequency selective surface filters for earth observation remote sensing instruments," IEEE Transactions on Terahertz Science and Technology, vol. 1, no. 2, pp. 450-461, 2011.

[21] T. S. Chu, M. J. Gans, and W. E. Legg, "Quasi-optical polarization diplexing of microwaves," Bell Labs Technical Journal, vol. 54, no. 10, pp. 1665-1680, 1975. 
[22] A. E. Costley, K. H. Hursey, G. F. Neill, and J. M. Ward, "Free-standing fine-wire grids: their manufacture, performance, and use at millimeter and submillimeter wavelengths," Journal of the Optical Society of America, vol. 67, no. 7, pp. 979-981, 1977.

[23] R. Ulrich, K. F. Renk, and L. Genzel, “Tunable submillimeter interferometers of the fabry-perot type," IEEE Transactions on Microwave Theory and Techniques, vol. 11, no. 5, pp. 363-371, 1963.

[24] I. Yamada, K. Takano, M. Hangyo, M. Saito, and W. Watanabe, "Terahertz wire-grid polarizers with micrometer-pitch Al gratings," Optics Letters, vol. 34, no. 3, pp. 274-276, 2009.

[25] J. R. Middendorf, J. S. Cetnar, J. Owsley, and E. R. Brown, "High fillfactor substrate-based wire-grid polarizers with high extinction ratios," IEEE Transactions on Terahertz Science and Technology, vol. 4, no. 3, pp. 376-382, 2014.

[26] C. Rieckmann, R. Dubrovka, and C. G. Parini, "Numerical and experimental investigation on the performance of planar millimetre-wave frequency selective surfaces," in Proceedings of The European Conference on Antennas and Propagation: EuCAP 2006 (ESA SP-626), pp. 89-91, Nice, France, 2006.

[27] A. Yamsiri and R. S. Donnan, "Validation of QO design tool for analysing aberration caused by FSS," in 2008 Loughborough Antennas and Propagation Conference (LAPC 2008), pp. 305308, Loughborough, UK, 2008.

[28] R. Zentner, M. Dadic, Z. Sipus, and J. Bartolic, "Time domain analysis of mutual coupling measurements between stacked patches," in 17th International Conference on Applied Electromagnetics and Communications, pp. 370-373, Dubrovnik, Croatia, 2003.

[29] J. Yu, X. Liu, Y. Yao et al., "The design and manufacture of a high frequency CATR," in Millimeter Waves and THz Technology Workshop (UCMMT), 2013 6th UK, Europe, China, pp. 1-2, Rome, Italy, 2013.

[30] X. Chen and X. Liu, "Terahertz antennas and measurement," in Handbook of Antenna Technologies, Springer, Singapore, 2015.

[31] Z. Lu, X. Liu, H. Wang, Y. Yao, X. Chen, and J. Yu, "Numerical and experimental verification of a trireflector compact antenna test range in the terahertz band," Microwave and Optical Technology Letters, vol. 57, no. 7, pp. 1686-1689, 2015.

[32] S. Gregson, J. Mccormick, and C. Parini, Principles of planar near-field antenna measurements, IET Electromagnetic Waves, The Institution of Engineering and Technology, London UK, 2007.

[33] W. D. Burnside and I. J. Gupta, "A method to reduce stray signal errors in antenna pattern measurements," IEEE Transactions on Antennas and Propagation, vol. 42, no. 3, pp. 399405, 1994.

[34] M. Thumm, A. Jacobs, and M. Sorolla Ayza, "Design of short highpower $\mathrm{TE}_{11}-\mathrm{HE}_{11}$ mode converters in highly overmoded corrugated waveguides," IEEE Transactions on Microwave Theory and Techniques, vol. 39, no. 2, pp. 301-309, 1991.

[35] P. J. B. Clarricoats and A. D. Olver, Corrugated Horns for Microwave Antennas, vol. 27, no. 27, 1984, Peter Peregrinus Ltd, London, UK, 1984.

[36] C. Dragone, "Reflection, transmission, and mode conversion in a corrugated feed," Bell Labs Technical Journal, vol. 56, no. 6, pp. 835-867, 1977.
[37] C. A. Balanis, Antenna Theory: Analysis and Design, Harper and Row, 1982.

[38] P. A. S. Cruickshank, D. R. Bolton, D. A. Robertson, and R. J. Wylde, "Reducing standing waves in quasi-optical systems by optimal feedhorn design," in Joint 32nd International Conference on Infrared and Millimeter Waves, 2007 and the 2007 International Conference on Terahertz Electronics. IrmmwThz, pp. 941-942, Cardiff, UK, 2007.

[39] X. Liu, Y. Mai, H. Su et al., "Design of tri-reflector compact antenna test range for millimetre/sub-millimetre wave and thz antenna measurement," in 2011 International Workshop on Antenna Technology (iWAT), pp. 144-147, Hong Kong, China, 2011.

[40] J. Yu and X. Chen, Millimeter Wave and Terahertz Antenna Measurement Technique, Science Press, 2015.

[41] J. E. Hansen, Spherical Near-field Antenna Measurements, IET Electromagnetic Waves Series 26, Peter Peregrinus Ltd, London, United Kingdom, 1988.

[42] G. B. Arfken, H. J. Weber, and L. Ruby, "Mathematical methods for physicists, 4th ed," American Journal of Physics, vol. 64, no. 7, p. 959, 1996.

[43] C. C. Chen, "Transmission of microwave through perforated flat plates of finite thickness," IEEE Transactions on Microwave Theory and Techniques, vol. 21, no. 1, pp. 1-6, 1973.

[44] R. E. Collin and J. Brown, "The design of quarter-wave matching layers for dielectric surfaces," Proceedings of the IEE Part C: Monographs, vol. 103, no. 3, pp. 153-158, 1956.

[45] A. D. Olver, "Compact antenna test ranges," in The Seventh International Conference on Antennas and Propagation (ICAP 1991), vol. 1, pp. 99-108, York, UK, 1991.

[46] J. Hartmann, J. Habersack, H. J. Steiner, J. Lemanczyk, and P. D. Maagt, "Measurement of large test objects in compensated compact ranges up to $500 \mathrm{GHz}$," in 25th Antenna Workshop on Satellite Antenna Technology ESA/ESTEC, Noordwijk, The Netherlands, 2002. 


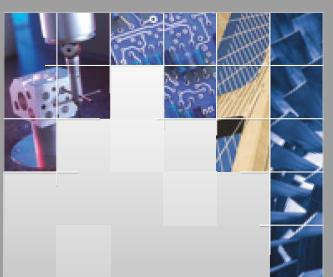

\section{Enfincering}
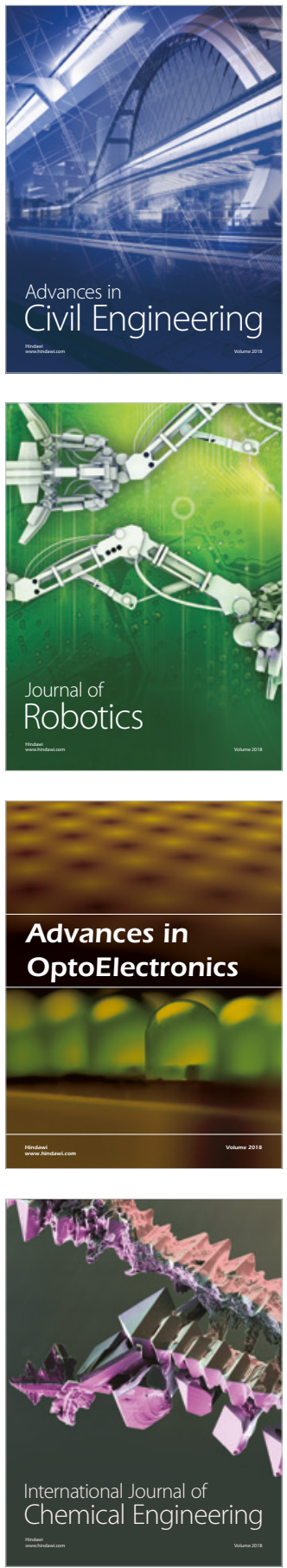

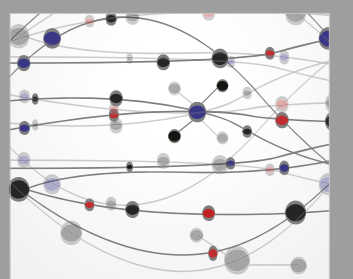

\section{Rotating \\ Machinery}

The Scientific World Journal

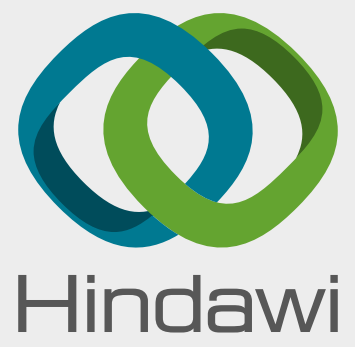

Submit your manuscripts at

www.hindawi.com
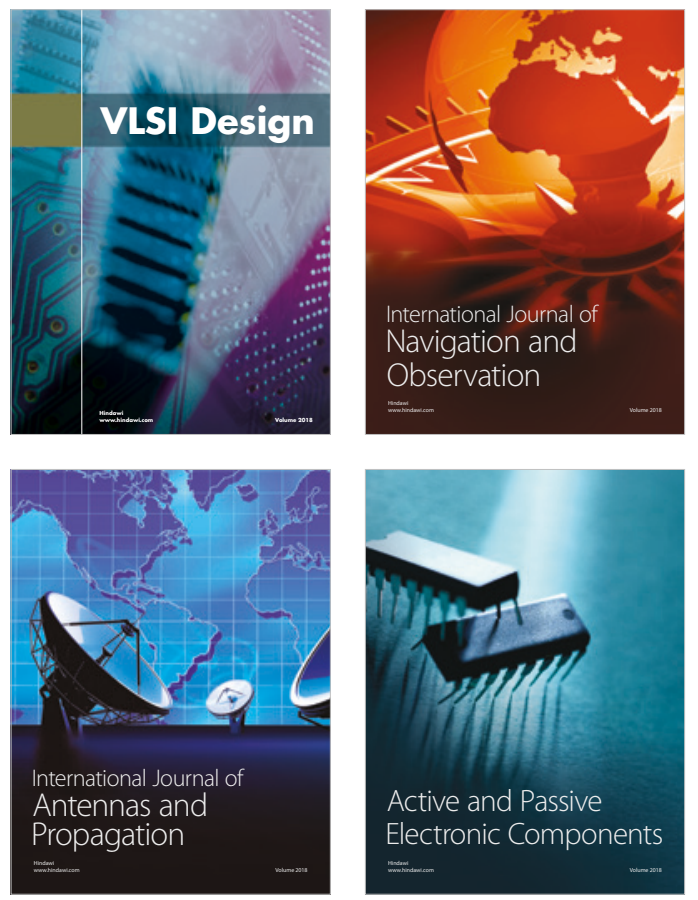
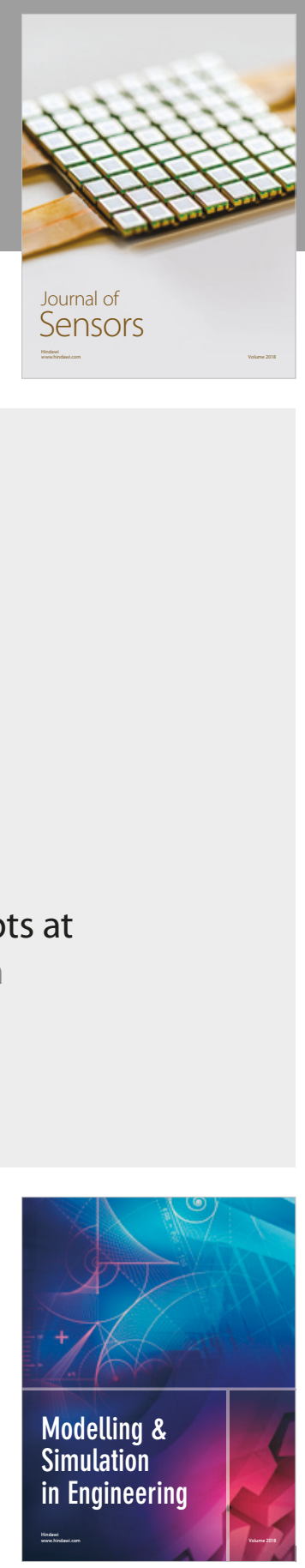

\section{Advances \\ Multimedia}
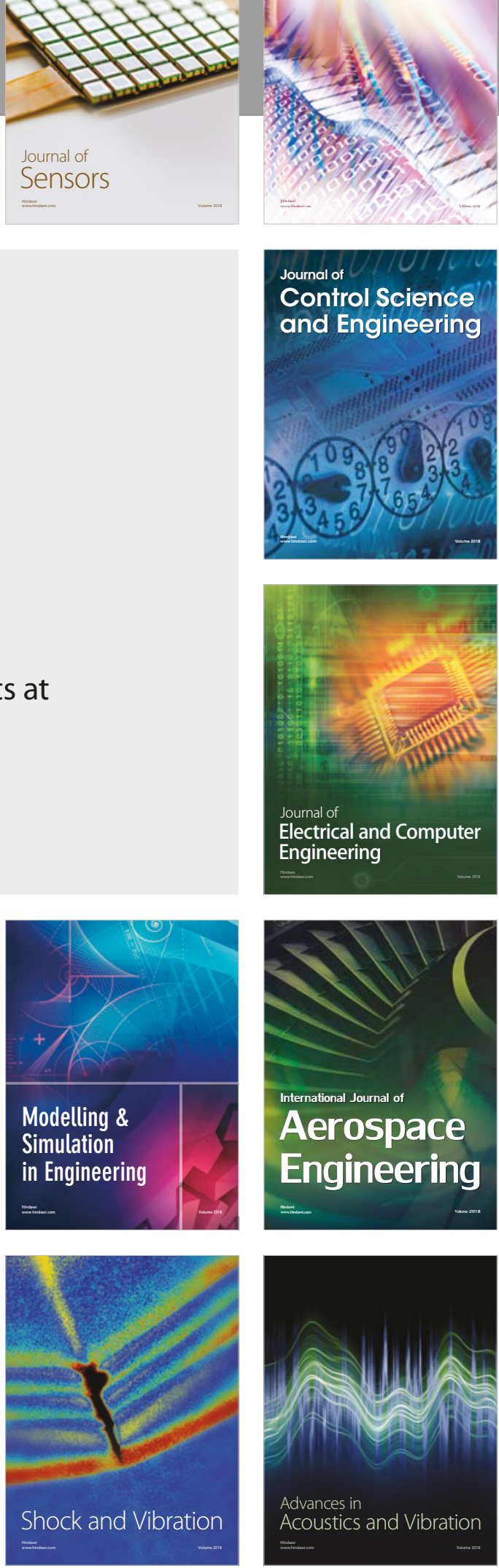